\title{
Turbulent flows as generalized Kelvin-Voigt materials: modeling and analysis
}

\author{
Cherif Amrouche ${ }^{1}$, Luigi C. Berselli ${ }^{2}$, Roger Lewandowski ${ }^{3}$, and Dinh Duong \\ Nguyen $^{4}$ \\ ${ }^{1}$ Laboratoire de Mathématiques et leurs Applications, UMR CNRS 5142, \\ Université de Pau et des Pays de l'Adour, France, E-mail: \\ cherif.amrouche@univ-pau.fr \\ ${ }^{2}$ Università di Pisa, Dipartimento di Matematica, Via Buonarroti 1/c, I-56127 \\ Pisa, Italy, E-mail: luigi.carlo.berselli@unipi.it \\ ${ }^{3,4}$ IRMAR, UMR CNRS 6625, University of Rennes 1 and FLUMINANCE Team, \\ INRIA Rennes, France, E-mail: Roger.Lewandowski@univ-rennes1.fr, \\ dinh-duong.nguyen@univ-rennes1.fr
}

\begin{abstract}
We model a 3D turbulent fluid, evolving toward a statistical equilibrium, by adding to the equations for the mean field $(\mathbf{v}, p)$ a term like $-\alpha \nabla \cdot\left(\ell(\mathbf{x}) D \mathbf{v}_{t}\right)$. This is of the Kelvin-Voigt form, where the Prandtl mixing length $\ell$ is not constant and vanishes at the solid walls. We get estimates for velocity $\mathbf{v}$ in $L_{t}^{\infty} H_{x}^{1} \cap W_{t}^{1,2} H_{x}^{1 / 2}$, that allow us to prove the existence and uniqueness of a regular-weak solutions $(\mathbf{v}, p)$ to the resulting system, for a given fixed eddy viscosity. We then prove a structural compactness result that highlights the robustness of the model. This allows us to pass to the limit in the quadratic source term in the equation for the turbulent kinetic energy $k$, which yields the existence of a weak solution to the corresponding Reynolds Averaged Navier-Stokes system satisfied by $(\mathbf{v}, p, k)$.
\end{abstract}

Key words : Fluid mechanics, Turbulence models, Navier-Stokes Equations, Turbulent Kinetic Energy.

2010 MSC: 76D05, 35Q30, 76F65, 76D03, 35Q30.

\section{Introduction}

The purpose of this paper is to model incompressible turbulent flows as generalized viscoelastic materials involving the Prandtl mixing length $\ell$ (see in [24]), to show the existence and uniqueness of regular-weak solutions to the resulting system of Partial Differential Equations (PDE),

$$
\left\{\begin{array}{r}
\mathbf{v}_{t}-\alpha \nabla \cdot\left(\ell(\mathbf{x}) D \mathbf{v}_{t}\right)+\nabla \cdot(\mathbf{v} \otimes \mathbf{v})-\nu \Delta \mathbf{v}-\nabla \cdot\left(\nu_{\text {turb }} D \mathbf{v}\right)+\nabla p=\mathbf{f}, \\
\nabla \cdot \mathbf{v}=0
\end{array}\right.
$$


for a given turbulent viscosity (eddy viscosity) $\nu_{\text {turb }}$. We then study the existence weak solutions to the corresponding NSTKE $^{1}$ system,

$$
\left\{\begin{array}{l}
\mathbf{v}_{t}-\alpha \nabla \cdot\left(\ell(\mathbf{x}) D \mathbf{v}_{t}\right)+\nabla \cdot(\mathbf{v} \otimes \mathbf{v})-\nu \Delta \mathbf{v}-\nabla \cdot\left(\nu_{\text {turb }}(k) D \mathbf{v}\right)+\nabla p=\mathbf{f} \\
\nabla \cdot \mathbf{v}=0 \\
k_{t}+\mathbf{v} \cdot \nabla k-\nabla \cdot\left(\mu_{\text {turb }}(k) \nabla k\right)=\nu_{\text {turb }}(k)|D \mathbf{v}|^{2}-(\ell+\eta)^{-1} k \sqrt{|k|}
\end{array}\right.
$$

where, to fix the notation,

- $\mathbf{v}$ is the mean velocity ${ }^{2}, \mathbf{v}_{t}=\frac{\partial \mathbf{v}}{\partial t}$;

- $D \mathbf{v}=\frac{1}{2}\left(\nabla \mathbf{v}+\nabla \mathbf{v}^{t}\right)$ is the deformation stress;

- $p$ is the mean pressure;

- $k$ is the Turbulent Kinetic Energy (TKE);

- $\nu>0$ is the kinematic viscosity, $\nu_{\text {turb }}$ the eddy viscosity;

- $\mu_{t}$ is the eddy diffusion and $\eta>0$ is a small constant;

- the length scale $\alpha$ is that of the boundary layer, given by the relation

$$
\alpha=\frac{\nu}{u_{\star}}
$$

here $u_{\star}$ is the so called friction velocity (see [5]);

- $\mathbf{f}$ is a given source term.

As usual, the systems are set in a bounded Lipschitz domain $\Omega \subset \mathbb{R}^{3}$. The mixing length $\ell=\ell(\mathbf{x}) \geq 0$ is defined over $\Omega$ and, according to well known physical laws (see (2.5) and (2.6) below), $\ell \in C^{1}(\bar{\Omega})$ and vanishes at the boundary $\Gamma=\partial \Omega$ as follows:

$$
\ell(\mathbf{x}) \simeq d(\mathbf{x}, \Gamma)=\rho(\mathbf{x}), \quad \text { when } \quad \mathbf{x} \rightarrow \Gamma, \mathbf{x} \in \Omega,
$$

where $d(\mathbf{x}, \Gamma)$ denotes the distance of the point $\mathbf{x}$ from the boundary.

Model (1.1) is close to viscoelastic materials models, given by the Kelvin-Voigt relation:

$$
\boldsymbol{\sigma}=E \boldsymbol{\varepsilon}+\eta \varepsilon_{t}
$$

where $\boldsymbol{\sigma}$ denotes the Cauchy stress tensor and $\varepsilon$ the strain-rate tensor. In this case, $E$ is the modulus of elasticity and $\eta$ the viscosity (see for instance Germain [8] or Gurtin [10]). In fluid mechanics, $\varepsilon=D \mathbf{v}$, and this model is used to describe some non Newtonian fluids, such as lubricants. For such flows, the law (1.5) becomes

$$
\boldsymbol{\sigma}=-p \operatorname{Id}+\mu D \mathbf{v}+\gamma^{2} D \mathbf{v}_{t}
$$

that yields the incompressible Navier-Stokes-Voigt equations:

$$
\left\{\begin{array}{r}
\mathbf{v}_{t}-\gamma^{2} \Delta \mathbf{v}_{t}+\nabla \cdot(\mathbf{v} \otimes \mathbf{v})-\nu \Delta \mathbf{v}+\nabla p=\mathbf{f}, \\
\nabla \cdot \mathbf{v}=0 .
\end{array}\right.
$$

\footnotetext{
${ }^{1}$ RANS $=$ Reynolds Averaged Navier-Stokes. NSTKE $=$ Navier-Stokes-Turbulent-Kinetic-Energy. NSTKE model is a specific RANS model.

${ }^{2}$ Usually, the mean velocity is denoted by $\overline{\mathbf{v}}$. Throughout the paper we omit the over-line for simplicity, except in Section 2, devoted to turbulence modelling.
} 
Mathematical investigations about system (1.6) were first carried out by A. Oskolkov, who proved the existence and uniqueness of weak and strong solutions in some particular sense, see [22, 23]. Then, several mathematical problems raised by (1.6) have been studied by Titi et al. [11, 15, 25], making a clear relation between Navier-Stokes-Voigt and turbulence modeling. In addition, in [12] Larios \& Titi showed the connection between the Navier-Stokes-Voigt equations and the simplified Bardina's model introduced by Layton \& Lewandowski [13], designed as a Large-Eddy simulation model. In Berselli, Kim, and Rebholz [3] an interpretation of the Navier-Stokes-Voigt equations in terms of approximate deconvolution models is also given.

In this paper we connect the Prandtl-Smagorinsky's model to the Turbulent Kinetic Energy (TKE) model to calculate the eddy viscosity $\nu_{\text {turb }}$. To make it clear, let $\boldsymbol{\sigma}^{(\mathrm{R})}$ denotes the Reynolds stress. We will show how, combining the energy inequality with the equation satisfied by $k$ (without any closure assumption), we are led to set -in certain specific regimes, such as the convergence to stable statistical states see (2.13)- the following constitutive law

$$
\boldsymbol{\sigma}^{(\mathrm{R})}=-\alpha \ell D \mathbf{v}_{t}-\nu_{\text {turb }} D \mathbf{v}+\frac{2}{3} k \mathrm{Id},
$$

instead of the usual one

$$
\boldsymbol{\sigma}^{(\mathrm{R})}=-\nu_{\text {turb }} D \mathbf{v}+\frac{2}{3} k \mathrm{Id} .
$$

This yields the PDE system (1.1) including the term $-\alpha \nabla \cdot\left(\ell D \mathbf{v}_{t}\right)$, and then also the NSTKE system (1.2) after having performed the usual closure procedure about $k$, where $\nu_{\text {turb }}=\nu_{\text {turb }}(k)=\ell \sqrt{k}$.

Turning to the analysis of the systems, we observe that according to assumption (1.4) about the mixing length $\ell$, the additional generalized Kelvin-Voigt term $-\alpha \nabla \cdot\left(\ell D \mathbf{v}_{t}\right)$ enforces for the equations a natural functional structure in the space $H^{1 / 2}(\Omega)=\left[H^{1}(\Omega), L^{2}(\Omega)\right]_{1 / 2}$, cf. Lions \& Magenes [19], which is a critical scaling-invariant space for the Navier-Stokes equations. In particular, we obtain for the velocity sharp estimates in $W^{1,2}\left(0, T ; H^{1 / 2}(\Omega)^{3}\right)$, as well as in $L^{\infty}\left(0, T ; H_{0}^{1}(\Omega)^{3}\right)$. We are then able to prove the existence and uniqueness of regular-weak solution to (1.1) (see Theorem 4.1 and Remark 4.5).

However, we believe that the most interesting result of this paper is the compactness result we prove in Lemma 5.1. We consider an eddy viscosities sequence $\left(\nu_{\text {turb }}^{n}\right)_{n \in \mathbf{N}}$ which is bounded in $L^{\infty}\left(\left[0, \infty[\times \Omega)\right.\right.$ and in addition converges a.e. to $\nu_{\text {turb }}$ in $[0, \infty[\times \Omega$ as $n \rightarrow \infty$. We also show that the corresponding regular-weak sequence of solution $\left(\mathbf{v}^{n}\right)_{n \in \mathbb{N}}$ converges, in some sense, to the regular-weak solution $\mathbf{v}$ of the limit problem with $\nu_{\text {turb }}$ as eddy viscosity. Moreover, we get the convergence of the energies, that is $\nu_{\text {turb }}^{n}\left|D \mathbf{v}^{n}\right|^{2} \rightarrow \nu_{\text {turb }}|D \mathbf{v}|^{2}$ in the sense of the measures.

This compactness result allows us to prove the existence of a solution to the NSTKE-Voigt system (1.2) (see Theorem 5.1 below). We stress that the usual system coupling $\mathbf{v}, p$. and $k$ only yields a variational inequality for $k$ when passing to the limit in the equations, because of the lack of strong convergence of the energies (see $[5,16])$. This observation makes Theorem 5.1 a very interesting and original result.

Plan of the paper. The paper is organized as follows: Section 2 is devoted to modeling and to explain the motivations for the systems of PDE we study. Then, in Section 3 we use functional analysis and interpolation theory to provide estimates in various spaces, especially in $H^{1 / 2}(\Omega)$. The proof of the existence and uniqueness results for the generalized Navier-Stokes-Voigt equations (1.1) and (4.1) is developed in Section 4. Finally, the compactness result and analysis of the NSTKE-Voigt system is performed in Section 5. 


\section{Kelvin-Voigt modeling for turbulent flows}

In this section (and only in this section) $\mathbf{v}$ and $p$ denote the velocity and pressure of the fluid respectively (and not the mean fields unlike in the rest of the paper). Hence, the couple $(\mathbf{v}, p)$ solves the Navier-Stokes equations,

$$
\left\{\begin{aligned}
\mathbf{v}_{t}+\nabla \cdot(\mathbf{v} \otimes \mathbf{v})-\nu \Delta \mathbf{v}+\nabla p & =\mathbf{f}, \\
\nabla \cdot \mathbf{v} & =0 .
\end{aligned}\right.
$$

We first recall some results about basic turbulence modeling to derive the equation for the mean $\overline{\mathbf{v}}$ and to define the Reynolds stress $\boldsymbol{\sigma}^{(\mathrm{R})}$. Then, we show how -when simultaneously using the Prandtl-Smagorinsky and the turbulent kinetic energy models and the equation satisfied by the TKE- we get the additional term $-\alpha \nabla \cdot\left(\ell D \overline{\mathbf{v}}_{t}\right)$ in the equation for $\overline{\mathbf{v}}$ in specific regimes, such as the convergence to a statistical equilibrium (see Remark 2.2).

We wish to mention that a very close modeling process has been previously performed in Yao, Layton, and Zhao [26]. The latter paper gave to us some inspiration for this modelling procedure we develop here. One main difference is that we study the TKE equations, while in their paper, Yao, Layton, and Zhao considered a rotational structure, without involving the equation for the turbulent kinetic energy. Moreover, they were looking at back-scatter terms, so that our point of view and interpretation are -at the very end-rather different.

\subsection{Recalls of basic turbulence modeling}

According to the Reynolds decomposition, $\mathbf{v}$ and $p$ are decomposed as the sum of their mean and fluctuation

$$
\mathbf{v}=\overline{\mathbf{v}}+\mathbf{v}^{\prime}, \quad \text { and } \quad p=\bar{p}+p^{\prime}
$$

where the mean filter is linear, commutes with any differential operator (namely $D \bar{\psi}=$ $\overline{D \psi}$ ), and it is idempotent (that is $\overline{\bar{\psi}}=\bar{\psi}$ ). From this, one gets the relation

$$
\overline{\mathbf{v} \otimes \mathbf{v}}=\overline{\mathbf{v}} \otimes \overline{\mathbf{v}}+\boldsymbol{\sigma}^{(\mathrm{R})},
$$

where the Reynolds stress $\boldsymbol{\sigma}^{(\mathrm{R})}$ is given by

$$
\boldsymbol{\sigma}^{(\mathrm{R})}=\overline{\mathbf{v}^{\prime} \otimes \mathbf{v}^{\prime}} .
$$

Therefore, applying the mean operator to the NSE (2.1) yields

$$
\left\{\begin{aligned}
\overline{\mathbf{v}}_{t}+\nabla \cdot(\overline{\mathbf{v}} \otimes \overline{\mathbf{v}})-\nu \Delta \overline{\mathbf{v}}+\nabla \cdot \boldsymbol{\sigma}^{(\mathrm{R})}+\nabla \bar{p} & =\overline{\mathbf{f}}, \\
\nabla \cdot \overline{\mathbf{v}} & =0 .
\end{aligned}\right.
$$

To "close" (2.2), one must express $\boldsymbol{\sigma}^{(\mathrm{R})}$ in terms of mean quantities. As we already said in the introduction, the Boussinesq assumption [4] yields

$$
\boldsymbol{\sigma}^{(\mathrm{R})}=-\nu_{\mathrm{turb}} D \overline{\mathbf{v}}+\frac{2}{3} k \mathrm{Id},
$$

where we recall that $\nu_{\text {turb }}$ is the eddy viscosity, $k=\frac{1}{2} \overline{\left|\mathbf{v}^{\prime}\right|^{2}}$ the turbulent kinetic energy (TKE), and $D \mathbf{v}=\frac{1}{2}\left(\nabla \mathbf{v}+\nabla \mathbf{v}^{T}\right)$ the deformation tensor.

The challenge in turbulence modelling is the determination of $\nu_{\text {turb }}$. In what follows, we combine the Prandtl-Smagorinsky's model with

$$
\nu_{\text {turb }}=\ell \sqrt{\alpha \ell}|D \overline{\mathbf{v}}|,
$$


where the boundary layer length scale $\alpha$ is given by (1.3), and the NSTKE model with $\nu_{\text {turb }}$ is given by

$$
\nu_{\text {turb }}=\ell \sqrt{k} \text {. }
$$

Dimensionless constants may be involved in the above equations. We have set them equal to 1 for the sake of simplicity. Both models involve the Prandtl mixing length $\ell$. In the case of a flow over a plate $\Omega=\mathbb{R}^{2} \times\{z>0\}$, one finds in Obukhov [21] the following law

$$
\ell=\ell(z)=\kappa z
$$

where $\kappa \in[0.35,0.42]$ is the Von Kármán constant. A more sophisticated formula, however very popular especially for the use in the computation of the turbulent channel flow, can be found in Van Driest [28]:

$$
\ell=\ell(z)=\kappa z\left(1-e^{-z / A}\right)
$$

where $A$ depends on the oscillations of the plate and $\nu$. Alternative formulas are provided in [18]. In all cases, $\ell$ satisfies the law (1.4).

\subsection{Modelling process}

We start from the natural energy inequality deduced from the equation (2.2) by usual integration by parts, at any time positive time $t^{3}$,

$$
\frac{1}{2} \frac{d}{d t}\|\overline{\mathbf{v}}(t)\|^{2}+\nu\|\nabla \overline{\mathbf{v}}(t)\|^{2}+\left\langle\nabla \cdot \boldsymbol{\sigma}^{(\mathrm{R})}, \overline{\mathbf{v}}(t)\right\rangle \leq\langle\overline{\mathbf{f}}(t), \overline{\mathbf{v}}(t)\rangle .
$$

We aim to evaluate the contribution of the term

$$
\mathscr{T}(t)=\left\langle\nabla \cdot \boldsymbol{\sigma}^{(\mathrm{R})}, \overline{\mathbf{v}}(t)\right\rangle,
$$

which will be deduced from the equation satisfied by $k$ (see [5, Sec. 4.4.1])

$$
\partial_{t} k+\overline{\mathbf{v}} \cdot \nabla k+\nabla \cdot \overline{e^{\prime} \mathbf{v}^{\prime}}=-\boldsymbol{\sigma}^{(\mathrm{R})}: \nabla \overline{\mathbf{v}}-\varepsilon+\overline{\mathbf{f}^{\prime} \cdot \mathbf{v}^{\prime}},
$$

where $e=k+e^{\prime}=\frac{1}{2}\left|\mathbf{v}^{\prime}\right|^{2}$ denotes the kinetic energy of the fluctuations, and $\varepsilon$ is the turbulent dissipation,

$$
\varepsilon:=\nu \overline{\left|D \mathbf{v}^{\prime}\right|^{2}} .
$$

Integrating formally this equation in space, leaving apart eventual boundary condition issues, leads to

$$
\frac{d}{d t} \int_{\Omega} k(t)=\mathscr{T}(t)-\int_{\Omega} \varepsilon(t)+\overline{\left\langle\mathbf{f}^{\prime}, \mathbf{v}^{\prime}\right\rangle}
$$

that we insert in the inequality (2.7) to obtain

$$
\left\langle\overline{\mathbf{v}}_{t}, \overline{\mathbf{v}}\right\rangle+\frac{d}{d t} \int_{\Omega} k+\nu\|\nabla \overline{\mathbf{v}}(t)\|^{2}+\int_{\Omega} \varepsilon(t) \leq\langle\overline{\mathbf{f}}(t), \overline{\mathbf{v}}(t)\rangle+\overline{\left\langle\mathbf{f}^{\prime}(t), \mathbf{v}^{\prime}(t)\right\rangle} .
$$

In order to eliminate the term $\frac{d}{d t} \int_{\Omega} k$ from (2.8), we enforce equality between the PrandtlSmagorinsky's model (2.3) and the NSTKE one (2.4), which leads to the closure equality

$$
k=\alpha \ell|D \overline{\mathbf{v}}|^{2} .
$$

\footnotetext{
${ }^{3}$ We use $\|\cdot\|$ for the $L^{2}$-norm in this section.
} 
Then, by using (2.9), we get the formal identity

$$
\frac{d}{d t} \int_{\Omega} k=\alpha \frac{d}{d t} \int_{\Omega} \ell|D \overline{\mathbf{v}}|^{2}=-\left\langle\alpha \nabla \cdot\left(\ell D \overline{\mathbf{v}}_{t}\right), \overline{\mathbf{v}}\right\rangle .
$$

Finally, we combine (2.8) with (2.10), which leads to the inequality

$$
\left\langle\overline{\mathbf{v}}_{t}-\alpha \nabla \cdot\left(\ell D \overline{\mathbf{v}}_{t}\right)-\nu \Delta \overline{\mathbf{v}}, \overline{\mathbf{v}}\right\rangle+\int_{\Omega} \varepsilon(t) \leq\langle\overline{\mathbf{f}}(t), \overline{\mathbf{v}}(t)\rangle+\overline{\left\langle\mathbf{f}^{\prime}(t), \mathbf{v}^{\prime}(t)\right\rangle} .
$$

Combining (2.7) to (2.11), suggests to put

$$
\boldsymbol{\sigma}^{(\mathrm{R})}=-\alpha \ell D \overline{\mathbf{v}}_{t}-\nu_{\text {turb }} D \overline{\mathbf{v}}+\frac{2}{3} k \mathrm{Id},
$$

and yields the following energy inequality

$$
\frac{1}{2} \frac{d}{d t}\left(\|\overline{\mathbf{v}}(t)\|^{2}+\alpha\|\sqrt{\ell} D \overline{\mathbf{v}}\|^{2}\right)+\nu\|\nabla \overline{\mathbf{v}}(t)\|^{2}+\left\|\sqrt{\nu_{\text {turb }}} D \overline{\mathbf{v}}\right\|^{2} \leq\langle\overline{\mathbf{f}}(t), \overline{\mathbf{v}}(t)\rangle .
$$

Comparing inequalities (2.12) and (2.11), we see that all this makes sense when:

$$
\left\|\sqrt{\nu_{\text {turb }}} D \overline{\mathbf{v}}\right\|^{2}+\overline{\left\langle\mathbf{f}^{\prime}(t), \mathbf{v}^{\prime}(t)\right\rangle} \leq\|\sqrt{\varepsilon(t)}\|^{2},
$$

and in this case the system satisfied by $\overline{\mathbf{v}}$ becomes

$$
\left\{\begin{aligned}
\overline{\mathbf{v}}_{t}-\alpha \nabla \cdot\left(\ell D \overline{\mathbf{v}}_{t}\right)+\nabla \cdot(\overline{\mathbf{v}} \otimes \overline{\mathbf{v}})-\nu \Delta \overline{\mathbf{v}}-\nabla \cdot\left(\nu_{\text {turb }} D \overline{\mathbf{v}}\right)+\nabla\left(\bar{p}+\frac{2}{3} k\right) & =\overline{\mathbf{f}}, \\
\nabla \cdot \overline{\mathbf{v}} & =0 .
\end{aligned}\right.
$$

Remark 2.1. When $\ell$ is constant and equal to $2 \alpha$ (to set ideas), and as $\nabla \cdot \overline{\mathbf{v}}_{t}=0$, we have $\alpha \nabla \cdot\left(\ell D \overline{\mathbf{v}}_{t}\right)=\alpha^{2} \Delta \overline{\mathbf{v}}_{t}$. Therefore, we get by this way the Kelvin-Voigt term involved in Equation (1.6).

Remark 2.2. Condition (2.13) asks for comments. To see if it may happen, let us take a constant source term $\mathbf{f}(t)=\mathbf{f}$, without turbulent fluctuation, which means $\mathbf{f}^{\prime}=\mathbf{0}$. In this case relation (2.13) simplifies to

$$
\left\|\sqrt{\nu_{\text {turb }}} D \overline{\mathbf{v}}\right\|^{2} \leq\|\sqrt{\varepsilon(t)}\|^{2} .
$$

The usual closed equation for $k$ is

$$
k_{t}+\overline{\mathbf{v}} \cdot \nabla k-\nabla \cdot\left(\mu_{\mathrm{turb}} \nabla k\right)=\nu_{\mathrm{turb}}|D \overline{\mathbf{v}}|^{2}-\varepsilon,
$$

giving, while ignoring possible boundary conditions,

$$
\frac{d}{d t} \int_{\Omega} k=\left\|\sqrt{\nu_{\mathrm{turb}}} D \overline{\mathbf{v}}\right\|^{2}-\|\sqrt{\varepsilon(t)}\|^{2} .
$$

Therefore, (2.14) indicates a decrease of TKE, which means a decrease of the turbulence, towards a laminar state, or a stable statistical equilibrium, such as a grid turbulence.

\section{Functional setting and estimate}

The analysis of system (1.1) yields immediately standard a priori estimates in $L_{t}^{\infty} L_{x}^{2}$ and $L_{t}^{2} H_{x}^{1}$, taking the solution itself as test function. The question is whether the Voigt term $-\alpha \nabla \cdot\left(\ell D \mathbf{v}_{t}\right)$ provides additional regularity. The issue is the degeneration of the mixing length $\ell$ at the boundary, according to (3.1) below. The purpose of this section is to derive from the interpolation theory a general estimate, that will enable us to show later that the term $-\alpha \nabla \cdot\left(\ell D \mathbf{v}_{t}\right)$ yields additional $W^{1,2}\left(0, T ; H^{1 / 2}\right)$ and $L^{\infty}\left(0, T ; H^{1}\right)$ regularity. 


\subsection{Framework and preliminaries}

As usual in mathematical fluid dynamics, we use the following spaces,

$$
\begin{aligned}
\mathcal{V} & =\left\{\boldsymbol{\varphi} \in \mathcal{D}(\Omega)^{3}, \nabla \cdot \boldsymbol{\varphi}=0 \text { in } \Omega\right\}, \\
H & =\left\{\mathbf{v} \in L^{2}(\Omega)^{3}, \nabla \cdot \mathbf{v}=0 \text { in } \Omega, \mathbf{v} \cdot \mathbf{n}=0 \text { on } \Gamma\right\}, \\
V & =\left\{\mathbf{v} \in H_{0}^{1}(\Omega)^{3}, \nabla \cdot \mathbf{v}=0 \text { in } \Omega\right\},
\end{aligned}
$$

and we recall that $\mathcal{V}$ is dense in $H$ and $V$ for their respective topologies [9]. Here $L^{2}(\Omega)$ and $H_{0}^{1}(\Omega)$ stand for the usual Lebesgue and Sobolev spaces.

Throughout the rest of the paper, the mixing length $\ell=\ell(\mathbf{x}) \in C^{1}(\bar{\Omega})$ is such that

$$
\left\{\begin{array}{l}
\forall K \subset \Omega, \quad K \text { compact, } \quad \inf _{K} \ell>0, \\
\ell(\mathbf{x}) \simeq d(\mathbf{x}, \Gamma)=\rho(\mathbf{x}), \quad \text { when } \quad \mathbf{x} \rightarrow \Gamma, \text { for } \mathbf{x} \in \Omega .
\end{array}\right.
$$

According to (3.1), we recall that

$$
H^{1 / 2}(\Omega)=\left[H^{1}(\Omega), L^{2}(\Omega)\right]_{1 / 2},
$$

and also

$$
H_{00}^{1 / 2}(\Omega)=\left[H_{0}^{1}(\Omega), L^{2}(\Omega)\right]_{1 / 2}=\left\{u \in H^{1 / 2}(\Omega), \text { s.t. } \quad \ell^{-1 / 2} u \in L^{2}(\Omega)\right\},
$$

cf. [19, Ch. 1]. In the following we will consider the following Hilbert space

$$
V_{1 / 2}=\left\{\mathbf{v} \in H^{1 / 2}(\Omega)^{3} ; \nabla \cdot \mathbf{v}=0 \text { in } \Omega \text { and } \mathbf{v} \cdot \mathbf{n}=0 \text { on } \Gamma\right\}
$$

equipped with the norm of $H^{1 / 2}(\Omega)^{3}$.

Finally, we know that when $\Omega$ is connected, the operator $D=\frac{\nabla+\nabla^{t}}{2}$ is well defined over $H^{s}(\Omega)^{3}$ whatever $s \geq 0$; next (see $[20]$ ),

$$
K:=\operatorname{Ker} D=\left\{\mathbf{v} \in H^{s}(\Omega)^{3} \text { s.t. } \exists(\mathbf{a}, \mathbf{b}) \in \mathbb{R}^{3} \times \mathbb{R}^{3} ; \mathbf{v}(\mathbf{x})=\mathbf{b} \times \mathbf{x}+\mathbf{a}\right\},
$$

and we recall the following Korn inequality

$$
\forall \mathbf{v} \in H^{1}(\Omega)^{3}, \quad\|\mathbf{v}\|_{H^{1}(\Omega)^{3} / K} \leq C\|D \mathbf{v}\|_{L^{2}(\Omega)^{9}},
$$

where for any given Banach space $B$ and any closed subspace $E \subset B, B / E$ denotes the quotient space. Moreover, for any $\mathbf{v} \in H_{0}^{1}(\Omega)^{3}$, we have $\|\mathbf{v}\|_{H^{1}(\Omega)^{3}} \leq C\|D \mathbf{v}\|_{L^{2}(\Omega)^{9}}$, because in this case the kernel $K$ is reduced to 0 .

\subsection{Main general estimate}

We deduce now the most relevant inequality, which derives from the generalized Voigt model when using the solution itself as test function.

Theorem 3.1. Let $\mathbf{v} \in \mathcal{D}^{\prime}(\Omega)^{3}$ such that $\sqrt{\ell} D \mathbf{v} \in L^{2}(\Omega)^{9}$. Then $\mathbf{v} \in H^{1 / 2}(\Omega)^{3}$ and there exists a constant $C=C(\Omega)$ such that

$$
\|\mathbf{v}\|_{H^{1 / 2}(\Omega)^{3} / K} \leq C\|\sqrt{\ell} D \mathbf{v}\|_{L^{2}(\Omega)^{9}} .
$$

In particular,

$$
W=\left\{\mathbf{v} \in H ; \sqrt{\ell} D \mathbf{v} \in L^{2}(\Omega)^{9}\right\} \hookrightarrow V_{1 / 2},
$$

with continuous embedding. 
Proof. We argue in two steps.

Step 1. Let $\mathbf{v} \in \mathcal{D}^{\prime}(\Omega)^{3}$ such that $\sqrt{\ell} D \mathbf{v} \in L^{2}(\Omega)^{9}$ and $\boldsymbol{\varphi} \in \mathcal{D}(\Omega)^{9}$. As $\mathbf{v} \in H_{\text {loc }}^{1}(\Omega)^{3}$, then we have

$$
|\langle D \mathbf{v}, \varphi\rangle|=\left|\int_{\Omega} \sqrt{\ell} D \mathbf{v}: \frac{\varphi}{\sqrt{\ell}}\right| \leq C\|\sqrt{\ell} D \mathbf{v}\|_{L^{2}(\Omega)^{9}}\|\varphi\|_{H_{00}^{1 / 2}(\Omega)^{9}} .
$$

Because of the density of $\mathcal{D}(\Omega)$ in $H_{00}^{1 / 2}(\Omega)$, this shows that $D \mathbf{v} \in\left[H_{00}^{1 / 2}(\Omega)^{9}\right]^{\prime}$ with the estimate

$$
\|D \mathbf{v}\|_{\left[H_{00}^{1 / 2}(\Omega)^{9}\right]^{\prime}} \leq C\|\sqrt{\ell} D \mathbf{v}\|_{L^{2}(\Omega)^{9}}
$$

Step 2. According to $[1,6]$, we have

$$
\forall \mathbf{v} \in L^{2}(\Omega)^{3}, \quad\|\mathbf{v}\|_{L^{2}(\Omega)^{3} / K} \leq C\|D \mathbf{v}\|_{H^{-1}(\Omega)^{9}} .
$$

Therefore, we deduce from classical interpolation theorems and from the following identities (see in [19]),

$$
\left[H^{1}(\Omega)^{3} / K, L^{2}(\Omega)^{3} / K\right]_{1 / 2}=H^{1 / 2}(\Omega)^{3} / K, \quad \text { and } \quad\left[L^{2}(\Omega), H^{-1}(\Omega)\right]_{1 / 2}=\left[H_{00}^{1 / 2}(\Omega)\right]^{\prime},
$$

the inequality

$$
\left.\|\mathbf{v}\|_{H^{1 / 2}(\Omega)^{3} / K} \leq C\|D \mathbf{v}\|_{\left[H_{00}^{1 / 2}(\Omega)^{9}\right.}\right]^{\prime} .
$$

Hence the estimate (3.3) follows by using (3.5) and obviously the embedding (3.4).

\section{Well-posedness for the generalized Navier-Stokes-Voigt equations}

In this section we start with the analysis of system (1.1) without any eddy viscosity, that means $\nu_{\text {turb }}=0$, both for simplicity of presentation and to highlight the role of the generalized Voigt term. The resulting system, called generalized Navier-Stokes-Voigt equations, is the following:

$$
\left\{\begin{aligned}
\mathbf{v}_{t}-\alpha \nabla \cdot\left(\ell D \mathbf{v}_{t}\right)+(\mathbf{v} \cdot \nabla) \mathbf{v}-\nu \Delta \mathbf{v}+\nabla p=\mathbf{f} & & \text { in }(0, T) \times \Omega, \\
\nabla \cdot \mathbf{v}=0 & & \text { in }(0, T) \times \Omega, \\
\left.\mathbf{v}\right|_{\Gamma}=0 & & \text { on }(0, T) \times \Gamma, \\
\mathbf{v}_{t=0}=\mathbf{v}_{0} & & \text { in } \Omega,
\end{aligned}\right.
$$

which is set in $Q_{T}=(0, T) \times \Omega$, where $\Omega$ is a given Lipschitz bounded domain in $\mathbb{R}^{3}$ with its boundary $\Gamma=\partial \Omega, T$ a fixed positive time ${ }^{4}$, and $\ell$ satisfies (1.4). The main results of this section are the existence and uniqueness of regular-weak solutions (see Definition 4.1 below), when the initial velocity $\mathbf{v}_{0} \in V$.

Throughout the rest of the paper, the $L^{2}$-norm of a given $u$ is simply denoted by $\|u\|,\|\cdot\|_{p}$ and $\|\cdot\|_{s, p}$ denote the standard $L^{p}$ and $W^{s, p}$ norms, respectively.

\footnotetext{
${ }^{4}$ Remind that when $\nabla \cdot \mathbf{v}=0$, then $\nabla \cdot(\mathbf{v} \otimes \mathbf{v})=(\mathbf{v} \cdot \nabla) \mathbf{v}$. We use either of these forms without necessarily warning, depending on the situation.
} 


\subsection{Strong solutions}

This aim of this subsection is to prove that given a finite time $T$, any strong (classical) solution $\mathbf{v}$ of (4.1) has natural bounds in $L^{\infty}(0, T ; V) \cap W^{1,2}\left(0, T ; V_{1 / 2}\right)$ derived from energy balances, showing that the term $-\alpha \nabla \cdot\left(\ell(\mathbf{x}) D \mathbf{v}_{t}\right)$-despite the degeneracy at the boundary- brings a strong regularizing effect on the system. In particular, the generalized Voigt term provides stronger a priori estimate when compared to the usual (non regularized) Navier-Stokes equations, since it allows to show bounds in critical scaling-invariant spaces $\grave{a}$ la Kato-Fujita. These estimates are essential for proving the existence result of the next subsection.

Following [14, 17], when considering $\mathbf{v}_{0} \in V \cap C(\bar{\Omega})^{3}$, we say that $(\mathbf{v}, p)$ is a strong solution to $(4.1)$ over $Q_{T}=[0, T] \times \Omega$, if

- $\forall \tau<T, \mathbf{v} \in C^{2}\left(Q_{\tau}\right)^{3}, p \in C^{1}\left(Q_{\tau}\right)$, and they satisfy the relations $((4.1)$, i), ii)) in the classical sense in $Q_{\tau}=[0, \tau] \times \Omega$,

- $\mathbf{v}(t, \cdot) \in C(\bar{\Omega})^{3}$ for all $t<T$, and $\mathbf{v}(t, \cdot)=0$ on $\Gamma$,

- $\mathbf{v}(t, \cdot)$ uniformly converges to $\mathbf{v}_{0}$ as $t \rightarrow 0^{+}$.

Remark 4.1. We frequently talk about the velocity $\mathbf{v}$ as a strong solution, without mentioning the pressure $p$. This means that we have implicitly projected the system over divergence-free vector fields by the Leray projector, which eliminates the pressure. The pressure can be recovered via the De Rham procedure (see e.g. Temam [27]).

Remark 4.2. We say that a strong solution $\mathbf{v}$ of (4.1) has a singularity at a given time $0<T^{\star}<\infty$ if $\|\mathbf{v}(t)\|_{\infty} \rightarrow \infty$ as $t \rightarrow T^{\star}, t<T^{\star}$. At this stage, we are not able to show that any strong solution has no singularity. We do not even know if there exist strong solutions, which is an open problem.

The estimates we get are based on the following non standard version of Gronwall's Lemma, the proof of which is carried out, e.g., in Emmrich [7].

Lemma 4.1. Let $\lambda \in L^{1}([0, T])$, with $\lambda(t) \geq 0$ for almost all $t \in[0, T]$, let $g \in C([0, T])$ be a non-decreasing function, and let $f \in L^{\infty}([0, T])$, such that $\forall t \in[0, T]$, it holds

$$
f(t) \leq g(t)+\int_{0}^{t} \lambda(s) f(s) d s
$$

Then, we have

$$
f(t) \leq g(t) \exp \left(\int_{0}^{t} \lambda(s) d s\right)
$$

In this subsection we assume that $\mathbf{f}(t)=\mathbf{f} \in C(\bar{\Omega})$ does not depend on $t$, and we denote by $F$ either $\|\mathbf{f}\|_{-1,2}^{2}$ or $\|\mathbf{f}\|^{2}$ so far non risk of confusion occurs, and $C$ denotes any constant (normally $C_{p}\|\mathbf{f}\| \leq\|\mathbf{f}\|_{-1,2}, C_{p}$ being the Poincaré's constant). Among many choices for the functional spaces of the source term (see also the discussion in the next subsection, where different choices are considered), this one has the advantage that it yields a clear and neat bound of the growth of the r.h.s in the estimates for statistical equilibrium.

The main result of this subsection is the following. 
Lemma 4.2. Let $\mathbf{v}$ be a strong solution of (4.1) in $Q_{T}=[0, T] \times \Omega$, for a given time $T$. Then, the following estimates hold true for all $s \in[0, T[$ :

$$
\begin{aligned}
& \|\mathbf{v}(s)\|_{1 / 2,2}^{2}+\nu \int_{0}^{s}\|\nabla \mathbf{v}(t)\|^{2} d t \leq C\left(\frac{F s}{\nu}+E(0)(\alpha, \ell)\right), \\
& \nu\|\nabla \mathbf{v}(s)\|^{2} \leq\left(\nu\left\|\nabla \mathbf{v}_{0}\right\|^{2}+F s\right) \exp \left\{\frac{C}{\alpha \nu^{2}}\left(\frac{F s}{\nu}+E(0)(\alpha, \ell)\right)\right\},
\end{aligned}
$$

and

$$
\begin{aligned}
& C \alpha \int_{0}^{s}\left\|\mathbf{v}_{t}(t)\right\|_{1 / 2,2}^{2} d t+\alpha \int_{0}^{s}\left\|\sqrt{\ell} D \mathbf{v}_{t}(t)\right\|^{2} d t \leq F s+\nu\left\|\nabla \mathbf{v}_{0}\right\|^{2} \\
& \quad+\frac{C}{\alpha \nu^{2}}\left(\nu\left\|\nabla \mathbf{v}_{0}\right\|^{2}+F T\right)\left(\frac{F s}{\nu}+E(0)(\alpha, \ell)\right) \exp \left\{\frac{C}{\alpha \nu^{2}}\left(\frac{F T}{\nu}+E(0)(\alpha, \ell)\right)\right\},
\end{aligned}
$$

where $2 E(0)(\alpha, \ell)=\left\|\mathbf{v}_{0}\right\|^{2}+\alpha\left\|\sqrt{\ell} D \mathbf{v}_{0}\right\|^{2}$. In particular, $\mathbf{v}$ has natural bounds in $L^{\infty}(0, T ; V)$ $\cap W^{1,2}\left(0, T ; V_{1 / 2}\right)$ and $\sqrt{\ell} D \mathbf{v}_{t} \in L^{2}\left(0, T ; L^{2}(\Omega)^{9}\right)$.

Proof. We take the dot product of $((4.1), \mathrm{i}))$ by $\mathbf{v}$. We integrate by parts and we use the identity $\langle(\mathbf{v} \cdot \nabla) \mathbf{v}, \mathbf{v}\rangle=0$. These calculations are justified because $\mathbf{v}$ is a strong solution, and this gives for any $s \in[0, T]$,

$$
\frac{1}{2} \frac{d}{d t}\left(\|\mathbf{v}(t)\|^{2}+\alpha\|\sqrt{\ell} D \mathbf{v}(t)\|^{2}\right)+\nu\|\nabla \mathbf{v}(t)\|^{2}=\langle\mathbf{f}(t), \mathbf{v}(t)\rangle \leq \frac{F}{2 \nu}+\frac{\nu}{2}\|\nabla \mathbf{v}(t)\|^{2},
$$

hence (4.2) follows after having integrated in time over $[0, s]$, by using (3.3), the fact that the norm of $V_{1 / 2}$ is that inherited from $H^{1 / 2}(\Omega)^{3}$, and $H^{1 / 2}(\Omega) \hookrightarrow L^{2}(\Omega)$ with continuous dense injection.

We next take the dot product of $((4.1), i))$ by $\mathbf{v}_{t}$. In this case the non-linear term brings a contribution in this new energy budget, given by

$$
\left\|\mathbf{v}_{t}(t)\right\|^{2}+\alpha\left\|\sqrt{\ell} D \mathbf{v}_{t}(t)\right\|^{2}+\frac{\nu}{2} \frac{d}{d t}\|\nabla \mathbf{v}(t)\|^{2}=\left\langle\mathbf{f}(t), \mathbf{v}_{t}(t)\right\rangle-\left\langle(\mathbf{v} \cdot \nabla) \mathbf{v}, \mathbf{v}_{t}\right\rangle(t),
$$

As we can estimate

$$
\left|\left\langle\mathbf{f}(t), \mathbf{v}_{t}(t)\right\rangle\right| \leq \frac{F}{2}+\frac{1}{2}\left\|\mathbf{v}_{t}(t)\right\|^{2},
$$

we obtain by using (3.3), keeping half of the contribution of the term $\alpha\left\|\sqrt{\ell} D \mathbf{v}_{t}(t)\right\|^{2}$,

$$
\frac{1}{2}\left\|\mathbf{v}_{t}(t)\right\|^{2}+C \alpha\left\|\mathbf{v}_{t}(t)\right\|_{1 / 2,2}^{2}+\frac{\alpha}{2}\left\|\sqrt{\ell} D \mathbf{v}_{t}(t)\right\|^{2}+\frac{\nu}{2} \frac{d}{d t}\|\nabla \mathbf{v}(t)\|^{2} \leq \frac{F}{2}+\left|\left\langle(\mathbf{v} \cdot \nabla) \mathbf{v}, \mathbf{v}_{t}\right\rangle(t)\right| .
$$

To deal with the nonlinear term, we use standard interpolation inequalities. The key of the process is the continuous embedding $H^{1 / 2}(\Omega) \hookrightarrow L^{3}(\Omega)^{3}$, which is the limit case. Therefore, we have

$$
\begin{aligned}
\left|\left\langle(\mathbf{v} \cdot \nabla) \mathbf{v}, \mathbf{v}_{t}\right\rangle(t)\right| & \leq\|\mathbf{v}(t)\|_{6}\|\nabla \mathbf{v}(t)\|\left\|\mathbf{v}_{t}(t)\right\|_{3} \\
& \leq C\|\nabla \mathbf{v}(t)\|^{2}\left\|\mathbf{v}_{t}(t)\right\|_{1 / 2,2} \\
& \leq \frac{1}{2 C \alpha}\|\nabla \mathbf{v}(t)\|^{4}+\frac{C \alpha}{2}\left\|\mathbf{v}_{t}(t)\right\|_{1 / 2,2}^{2}
\end{aligned}
$$

so that (4.5) becomes

$$
\left\|\mathbf{v}_{t}(t)\right\|^{2}+C \alpha\left\|\mathbf{v}_{t}(t)\right\|_{1 / 2,2}^{2}+\alpha\left\|\sqrt{\ell} D \mathbf{v}_{t}(t)\right\|^{2}+\nu \frac{d}{d t}\|\nabla \mathbf{v}(t)\|^{2} \leq F+\frac{1}{C \alpha}\|\nabla \mathbf{v}(t)\|^{4}
$$


In particular it follows from the above estimate that

$$
\nu \frac{d}{d t}\|\nabla \mathbf{v}(t)\|^{2} \leq F+\frac{1}{C \alpha}\|\nabla \mathbf{v}(t)\|^{4},
$$

that we integrate over $[0, s], s \in[0, T]$, so that

$$
\nu\|\nabla \mathbf{v}(s)\|^{2} \leq \nu\left\|\nabla \mathbf{v}_{0}\right\|^{2}+F s+\frac{1}{C \alpha} \int_{0}^{s}\|\nabla \mathbf{v}(t)\|^{4} d t .
$$

From there, Lemma 4.1 is applied on every time interval $[0, \tau]$ for $\tau<T$, with

$$
f(t)=\nu\|\nabla \mathbf{v}(t)\|^{2} \quad \text { and } \quad \lambda(t)=\frac{1}{C \alpha \nu}\|\nabla \mathbf{v}(t)\|^{2},
$$

both are in $L^{1}(0, \tau) \cap L^{\infty}(0, \tau)$ and $g(t)=\nu\left\|\nabla \mathbf{v}_{0}\right\|^{2}+F t$ which is a non decreasing function, which leads to

$$
\nu\|\nabla \mathbf{v}(s)\|^{2} \leq\left(\nu\left\|\nabla \mathbf{v}_{0}\right\|^{2}+F s\right) \exp \left\{\frac{1}{C \alpha \nu} \int_{0}^{s}\|\nabla \mathbf{v}(t)\|^{2} d t\right\},
$$

and yields (4.3) by using (4.2). Therefore, the inequality (4.4) is deduced from (4.6) combined with (4.2)-(4.3).

\subsection{Existence and uniqueness of regular-weak solutions}

We start by giving the definition of a "regular-weak solution" to the generalized NavierStokes-Voigt system (4.1). This definition is based on Lemma 4.2. We say "weak solution" since it is given by a weak formulation, "regular" since, because of Lemma 4.2, we will search for a solution in $L^{\infty}(0, T ; V) \cap W^{1,2}\left(0, T ; V_{1 / 2}\right)$. This space is considerably smaller than that involved in "standard" Leray-Hopf weak solutions to the Navier-Stokes equations (NSE) that are just in $L^{\infty}(0, T ; H) \cap L^{2}(0, T ; V)$. As we shall see it, regular weak solutions are unique and satisfy the energy equality, a fact which is still not known about weak solutions to the NSE.

Definition 4.1. We say that a function $\mathbf{v} \in L^{\infty}(0, T ; V) \cap W^{1,2}\left(0, T ; V_{1 / 2}\right)$ is a regularweak solution of the initial boundary value problem (4.1) if it holds true that

$$
\frac{d}{d t}[(\mathbf{v}, \boldsymbol{\phi})+\alpha(\ell D \mathbf{v}, D \boldsymbol{\phi})]+\nu(\nabla \mathbf{v}, \nabla \boldsymbol{\phi})+((\mathbf{v} \cdot \nabla) \mathbf{v}, \boldsymbol{\phi})=\langle\mathbf{f}, \boldsymbol{\phi}\rangle \quad \forall \boldsymbol{\phi} \in V,
$$

in the sense of $\mathcal{D}^{\prime}(0, T)$ and the initial datum is attained at least in the sense of $V_{1 / 2}$, that is

$$
\lim _{t \rightarrow 0^{+}}\left\|\mathbf{v}(t)-\mathbf{v}_{0}\right\|_{V_{1 / 2}}=0 .
$$

The main theorem we prove is the following one, showing the well-posedness of the system, globally in time. To fix the ideas and for the simplicity, we stay in a usual weak solutions framework by taking the source term $\mathbf{f}=\mathbf{f}(t)$ in the space $L^{2}\left(0, T ; H^{-1 / 2}(\Omega)^{3}\right)^{5}$. However, many variants can be considered, starting with $\mathbf{f} \in L^{2}\left(0, T ; V_{1 / 2}^{\prime}\right)$, or $\mathbf{f}(t)=\mathbf{f} \in L^{2}(\Omega)^{3}$ following the previous subsection, which does not change too much. An interesting case would be $\mathbf{f} \in L_{u l o c}^{2}\left(\mathbb{R}^{+} ; V_{1 / 2}^{\prime}\right)$, for which additional work remains to be done in the context of the long-time behavior (see [2]).

\footnotetext{
${ }^{5}$ Recall that $H^{-1 / 2}(\Omega)=\left[H_{0}^{1 / 2}(\Omega)\right]^{\prime}$ and be aware that $H^{-1 / 2}(\Omega) \subsetneq\left[H_{00}^{1 / 2}(\Omega)\right]^{\prime}$ with strict inclusion, see Lions-Magenes [19].
} 
Theorem 4.1. Let be given $\mathbf{v}_{0} \in V$ and $\mathbf{f} \in L^{2}\left(0, T ; H^{-1 / 2}(\Omega)^{3}\right)$. Then, there exists a unique regular-weak solution of the initial boundary value problem (4.1) in $[0, T]$, which satisfies the energy (of the model) equality for all $t \geq 0$,

$$
E(t)(\alpha, \ell)+\nu \int_{0}^{t}\|\nabla \mathbf{v}(s)\|^{2} d s=E(0)(\alpha, \ell)+\int_{0}^{t}\langle\mathbf{f}(s), \mathbf{v}(s)\rangle d s .
$$

where $E(t)(\alpha, \ell):=\frac{1}{2}\left(\|\mathbf{v}(t)\|^{2}+\alpha\|\sqrt{\ell} D \mathbf{v}(t)\|^{2}\right)$.

Proof. The proof follows by a standard Faedo-Galerkin approximation with suitable apriori estimates, compactness argument, and interpolation results. It is divided into the following four steps:

1) Construction of approximate solutions, locally in time;

2) Estimates;

3) Passing to the limit in the equations;

4) Energy balance and uniqueness.

Step 1. Construction of approximate solutions, locally in time. Let $\left\{\boldsymbol{\psi}_{n}\right\}_{n} \subset \mathcal{V}$ be a Hilbert basis of $V$ which we can suppose, without lack of generality, to be orthonormal in $H$ as well as orthogonal in $V$. We look for approximate Galerkin functions

$$
\mathbf{v}^{n}(t, x)=\sum_{j=1}^{n} c_{n j}(t) \boldsymbol{\psi}_{j}(x) \quad \text { for } n \in \mathbb{N},
$$

which has to solve the generalized Navier-Stokes-Voigt equations projected over $\mathbf{W}_{n}=$ $\operatorname{Span}\left(\boldsymbol{\psi}_{1}, \ldots, \boldsymbol{\psi}_{n}\right)$, that is

$$
\begin{aligned}
\frac{d}{d t}\left[\left(\mathbf{v}^{n}, \boldsymbol{\psi}_{m}\right)+\alpha\left(\ell D \mathbf{v}^{n}, D \boldsymbol{\psi}_{m}\right)\right]+\nu\left(\nabla \mathbf{v}^{n}, \nabla \boldsymbol{\psi}_{m}\right)+\left(\left(\mathbf{v}^{n} \cdot \nabla\right) \mathbf{v}^{n}, \boldsymbol{\psi}_{m}\right) & =\left\langle\mathbf{f}, \boldsymbol{\psi}_{m}\right\rangle, \\
\left(\mathbf{v}^{n}(0), \boldsymbol{\psi}_{m}\right) & =\left(\mathbf{v}_{0}, \boldsymbol{\psi}_{m}\right),
\end{aligned}
$$

for $m=1, \ldots, n$. The above problem is a Cauchy problem for a system of $n$-ordinary differential equations in the coefficients $c_{n m}(t)$. We define the following quantities for $j, l, m=1, \ldots, n$ :

$$
\begin{array}{rr}
\alpha_{j m}:=\alpha\left(\ell D \boldsymbol{\psi}_{j}, D \boldsymbol{\psi}_{m}\right), & \beta_{j m}:=\nu\left(\nabla \boldsymbol{\psi}_{j}, \nabla \boldsymbol{\psi}_{m}\right), \\
\gamma_{j l m}:=\left(\left(\boldsymbol{\psi}_{j} \cdot \nabla\right) \boldsymbol{\psi}_{l}, \boldsymbol{\psi}_{m}\right), & f_{m}(t):=\left\langle\mathbf{f}(t), \boldsymbol{\psi}_{m}\right\rangle,
\end{array}
$$

and we have a non-homogeneous system of ordinary differential equations with constant coefficients (which we write with the convention of summation over repeated indices)

$$
c_{n j}^{\prime}(t)\left(\delta_{j m}+\alpha_{j m}\right)+c_{n j}(t) \beta_{j m}+c_{n j}(t) c_{n l}(t) \gamma_{j l m}=f_{m}(t), \quad m=1, \ldots, n,
$$

where $\delta_{i j}$ denotes the standard Kronecker delta notation. The above system is not in normal form. In order to obtain a system for which we can apply the Cauchy-Lipschitz Theorem, we have to show that the matrix $\left(\delta_{j m}+\alpha_{j m}\right)$ can be inverted. Hence, since we work in a finite dimensional spaces it is enough to show that its kernel contains only the zero vector. So let $\boldsymbol{\xi}=\left(\xi_{1}, \ldots, \xi_{n}\right) \in \mathbb{R}^{n}$ be such that

$$
\left(\delta_{j m}+\alpha_{j m}\right) \xi_{j}=0 .
$$


Multiplying the above equation by $\xi_{m}$ and summing also over $m=1, \ldots, n$ leads to

$$
0=\|\boldsymbol{\xi}\|^{2}+\alpha(\ell \phi, \phi)=\|\boldsymbol{\xi}\|^{2}+\alpha(\sqrt{\ell} \phi, \sqrt{\ell} \phi) \geq\|\boldsymbol{\xi}\|^{2} \quad \text { with } \quad \phi:=\sum_{j=1}^{n} \xi_{j} D \boldsymbol{\psi}_{j},
$$

due to the facts that $\alpha>0$ and $\ell(\mathbf{x}) \geq 0$. Hence, this implies that $\boldsymbol{\xi} \equiv \mathbf{0}$, hence that the matrix $\left(\delta_{j m}+\alpha_{j m}\right)$ can be inverted. This allows to rewrite the system of ODEs for the coefficients $c_{n j}$ as follows

$c_{n j}^{\prime}(t)+c_{n j}(t)\left(\delta_{j m}+\alpha_{j m}\right)^{-1} \beta_{j m}+c_{n j}(t) c_{n l}(t)\left(\delta_{j m}+\alpha_{j m}\right)^{-1} \gamma_{j l m}=\left(\delta_{j m}+\alpha_{j m}\right)^{-1} f_{m}(t)$,

and to apply the basic theory of ordinary differential equations. Note that the coefficient from the right-hand side $f_{m}(t)=\left\langle\mathbf{f}(t), \boldsymbol{\psi}_{m}\right\rangle$ is not continuous but just $L^{2}(0, T)$, hence one has to resort to an extension of the Cauchy-Lipschitz theorem, with absolutely continuous functions, under Carathéodory hypotheses (see Walter [29]).

Since the system for the coefficients $c_{n j}(t)$ is nonlinear (quadratic) we obtain that there exists a unique solution $c_{n j}(t) \in W^{1,2}\left(0, T_{n}\right)$, for some $0<T_{n} \leq T$.

Step 2. Estimates. By taking $\mathbf{v}^{n}$ as test function, one gets the identity

$$
\frac{1}{2} \frac{d}{d t}\left(\left\|\mathbf{v}^{n}(t)\right\|^{2}+\alpha\left\|\sqrt{\ell} D \mathbf{v}^{n}(t)\right\|^{2}\right)+\nu\left\|\nabla \mathbf{v}^{n}(t)\right\|^{2}=\left\langle\mathbf{f}, \mathbf{v}^{n}\right\rangle
$$

from which it follows

$$
\frac{d}{d t}\left(\left\|\mathbf{v}^{n}(t)\right\|^{2}+\alpha\left\|\sqrt{\ell} D \mathbf{v}^{n}(t)\right\|^{2}\right)+\nu\left\|\nabla \mathbf{v}^{n}(t)\right\|^{2} \leq \frac{C_{P}}{\nu}\|\mathbf{f}\|_{-1 / 2,2}^{2},
$$

where $C_{P}=C_{P}(\Omega)$ is the Poincaré-type constant such that

$$
\|u\|_{1 / 2,2}^{2} \leq C_{P}\|\nabla u\|^{2} \quad \forall u \in H_{0}^{1}(\Omega) .
$$

Hence, integrating over $(0, t)$ for $t<T_{n}$ we get

$$
E^{n}(t)(\alpha, \ell)+\nu \int_{0}^{t}\left\|\nabla \mathbf{v}^{n}(s)\right\|^{2} d s \leq E^{n}(0)(\alpha, \ell)+\frac{C_{P}}{\nu} \int_{0}^{t}\|\mathbf{f}(s)\|_{-1 / 2,2}^{2} d s .
$$

where $E^{n}(t)(\alpha, \ell):=\left\|\mathbf{v}^{n}(t)\right\|^{2}+\alpha\left\|\sqrt{\ell} D \mathbf{v}^{n}(t)\right\|^{2}$. Next, we observe that since $\mathbf{v}^{n}(0) \rightarrow \mathbf{v}_{0}$ in $V$ and $0 \leq \ell \in C(\bar{\Omega})$, then it holds

$$
\alpha\left\|\sqrt{\ell} D \mathbf{v}^{n}(0)\right\|^{2} \leq \alpha \max _{x \in \bar{\Omega}} \ell(\mathbf{x})\left\|\nabla \mathbf{v}^{n}(0)\right\|^{2} \leq \alpha \max _{\mathbf{x} \in \bar{\Omega}} \ell(\mathbf{x})\left\|\nabla \mathbf{v}_{0}\right\|^{2},
$$

which shows that, under the given assumptions on $\mathbf{v}_{0}$ and $\mathbf{f}$ the r.h.s of (4.9) can be bounded independently of $n \in \mathbb{N}$ and consequently, a standard continuation argument proves in fact that $T_{n}=T$. Moreover, it also holds

$$
\mathbf{v}^{n} \in L^{\infty}(0, T ; H) \cap L^{2}(0, T ; V) \text { and } \quad \sqrt{\ell} D \mathbf{v}^{n} \in L^{\infty}\left(0, T ; L^{2}(\Omega)^{9}\right),
$$

with norms bounded uniformly in $n \in \mathbb{N}$. Therefore, according to Theorem 3.1, we also obtain

$$
\left\|\mathbf{v}^{n}(t)\right\|^{2}+\left\|\mathbf{v}^{n}(t)\right\|_{V_{1 / 2}}^{2}+\int_{0}^{t}\left\|\nabla \mathbf{v}^{n}(s)\right\|^{2} d s \leq C\left[\int_{0}^{t}\|\mathbf{f}(s)\|_{-1 / 2,2}^{2} d s+\left\|\mathbf{v}_{0}\right\|^{2}+\left\|\nabla \mathbf{v}_{0}\right\|^{2}\right],
$$


for a constant $C$ depending on $\nu, \alpha, \ell$ and $\Omega$. In addition, this inequality proves that

$$
\mathbf{v}^{n} \in L^{\infty}\left(0, T ; V_{1 / 2}\right)
$$

with bounds independent of $n \in \mathbb{N}$.

In order to give a proper meaning to the time derivative, we now use as test function $\mathbf{v}_{t}^{n}$, which is allowed, since it vanishes at the boundary and it is divergence-free. We get

$$
\left\|\mathbf{v}_{t}^{n}(t)\right\|^{2}+\alpha\left\|\sqrt{\ell} D \mathbf{v}_{t}^{n}(t)\right\|^{2}+\frac{\nu}{2} \frac{d}{d t}\left\|\nabla \mathbf{v}^{n}(t)\right\|^{2}=\left(\mathbf{f}, \mathbf{v}_{t}^{n}\right)-\left(\left(\mathbf{v}^{n} \cdot \nabla\right) \mathbf{v}^{n}, \mathbf{v}_{t}^{n}\right) .
$$

We estimate the r.h.s of (4.11), thanks to the Cauchy-Schwarz, Hölder, Young and Sobolev inequalities, which give us

$$
\left|\left(\mathbf{f}, \mathbf{v}_{t}^{n}\right)\right| \leq C_{\epsilon}\|\mathbf{f}\|_{-1 / 2,2}^{2}+\epsilon\left\|\mathbf{v}_{t}^{n}\right\|_{V_{1 / 2}}^{2},
$$

and

$$
\left|\left(\left(\mathbf{v}^{n} \cdot \nabla\right) \mathbf{v}^{n}, \mathbf{v}_{t}^{n}\right)\right| \leq\left\|\mathbf{v}^{n}\right\|_{6}\left\|\nabla \mathbf{v}^{n}\right\|\left\|\mathbf{v}_{t}^{n}\right\|_{3} \leq C\left\|\nabla \mathbf{v}^{n}\right\|^{2}\left\|\mathbf{v}_{t}^{n}\right\|_{V_{1 / 2}} \leq C_{\epsilon}\left\|\nabla \mathbf{v}^{n}\right\|^{4}+\epsilon\left\|\mathbf{v}_{t}^{n}\right\|_{V_{1 / 2}}^{2} .
$$

By the above inequalities we can absorb terms in the l.h.s, to obtain

$$
C\left\|\mathbf{v}_{t}^{n}(t)\right\|_{V_{1 / 2}}^{2}+\frac{\nu}{2} \frac{d}{d t}\left\|\nabla \mathbf{v}^{n}(t)\right\|^{2} \leq C_{\epsilon}\left[\|\mathbf{f}(t)\|_{-1 / 2,2}^{2}+\left\|\nabla \mathbf{v}^{n}(t)\right\|^{4}\right],
$$

for some $C_{\epsilon}=C(\ell, \alpha, \Omega)$. Integrating over $[0, s]$ for $s \in[0, T]$, one obtains

$$
\begin{aligned}
C \int_{0}^{s}\left\|\mathbf{v}_{t}^{n}(t)\right\|_{V_{1 / 2}}^{2} d t+\frac{\nu}{2}\left\|\nabla \mathbf{v}^{n}(s)\right\|^{2} \leq \frac{\nu}{2}\left\|\nabla \mathbf{v}^{n}(0)\right\|^{2}+C_{\epsilon} \int_{0}^{s}\|\mathbf{f}(t)\|_{-1 / 2,2}^{2} d t \\
+C_{\epsilon} \int_{0}^{s}\left\|\nabla \mathbf{v}^{n}(t)\right\|^{4} d t
\end{aligned}
$$

hence

$C \int_{0}^{s}\left\|\mathbf{v}_{t}^{n}(t)\right\|_{V_{1 / 2}}^{2} d t+\frac{\nu}{2}\left\|\nabla \mathbf{v}^{n}(s)\right\|^{2} \leq \frac{\nu}{2}\left\|\nabla \mathbf{v}_{0}\right\|^{2}+C_{\epsilon}\left(\int_{0}^{s}\|\mathbf{f}(t)\|_{-1 / 2,2}^{2} d t+\int_{0}^{s}\left\|\nabla \mathbf{v}^{n}(t)\right\|^{4} d t\right)$,

and in particular,

$$
\frac{\nu}{2}\left\|\nabla \mathbf{v}^{n}(s)\right\|^{2} \leq \frac{\nu}{2}\left\|\nabla \mathbf{v}_{0}\right\|^{2}+C_{\epsilon} \int_{0}^{s}\|\mathbf{f}(t)\|_{-1 / 2,2}^{2} d t+C_{\epsilon} \int_{0}^{s}\left\|\nabla \mathbf{v}^{n}(t)\right\|^{4} d t .
$$

We apply the Gronwall's lemma 4.1 to get

$$
\frac{\nu}{2}\left\|\nabla \mathbf{v}^{n}(s)\right\|^{2} \leq\left(\frac{\nu}{2}\left\|\nabla \mathbf{v}_{0}\right\|^{2}+C_{\epsilon} \int_{0}^{s}\|\mathbf{f}(t)\|_{-1 / 2,2}^{2} d t\right) \exp \left\{C_{\epsilon} \int_{0}^{s}\left\|\nabla \mathbf{v}^{n}(t)\right\|^{2} d t\right\},
$$

and the r.h.s of (4.13) is bounded uniformly in $n$ due the a priori estimate (4.10). This proves that

$$
\mathbf{v}^{n} \in L^{\infty}(0, T ; V),
$$

from which we also deduce by using (4.12) that

$$
\mathbf{v}_{t}^{n} \in L^{2}\left(0, T ; V_{1 / 2}\right), \quad \text { and therefore by }(4.10) \quad \mathbf{v}^{n} \in W^{1,2}\left(0, T ; V_{1 / 2}\right),
$$


with uniform bounds in $n \in \mathbb{N}$. Beside estimates in $V_{1 / 2}$, it is important to stress that with the same track, starting from (4.11) as in the proof of Lemma 4.2, we also have

$$
\sqrt{\ell} D \mathbf{v}_{t}^{n} \in L^{2}\left(0, T ; L^{2}(\Omega)^{9}\right),
$$

again with uniform bound in $n \in \mathbb{N}$.

Step 3. Passing to the limit in the approximate equations. By the uniform bounds above and standard compactness results there exists $\mathbf{v} \in W^{1,2}\left(0, T ; V_{1 / 2}\right) \cap L^{\infty}(0, T ; V)$ and a sub-sequence (relabelled as $\mathbf{v}^{n}$ ) such that

$$
\left\{\begin{array}{cll}
\mathbf{v}^{n} \stackrel{*}{\rightarrow} \mathbf{v} & \text { in } & L^{\infty}(0, T ; V), \\
\sqrt{\ell} D \mathbf{v}^{n} \stackrel{*}{ } \sqrt{\ell} D \mathbf{v} & \text { in } \quad L^{\infty}\left(0, T ; L^{2}(\Omega)^{9}\right), \\
\mathbf{v}^{n} \rightarrow \mathbf{v} & \text { in } \quad L^{p}(0, T ; V) \quad \text { for all } 1<p<\infty \\
\mathbf{v}_{t}^{n} \rightarrow \mathbf{v}_{t} & \text { in } \quad L^{2}\left(0, T ; V_{1 / 2}\right), \\
\sqrt{\ell} D \mathbf{v}_{t}^{n} \rightarrow \sqrt{\ell} D \mathbf{v}_{t} & \text { in } \quad L^{2}\left(0, T ; L^{2}(\Omega)^{9}\right),
\end{array}\right.
$$

To get strong convergence in appropriate spaces, we use the Aubin-Lions compactness lemma (see [27]) with the triple

$$
V \hookrightarrow V_{3 / 4} \hookrightarrow V_{1 / 2},
$$

where $V_{3 / 4}=[V, H]_{3 / 4}$, each embedding being dense and continuous. Moreover, since $\Omega$ is bounded by the Rellich-Kondrachov Theorem, these embeddings are also compact. Therefore, the sequence $\left(\mathbf{v}^{n}\right)_{n \in \mathbb{N}}$ is (pre)compact in $L^{2}\left(0, T ; V_{3 / 4}\right)$ and (up to a sub-sequence)

$$
\mathbf{v}^{n} \rightarrow \mathbf{v} \quad \text { in } \quad L^{2}\left(0, T ; V_{3 / 4}\right)
$$

which implies in particular strong convergence in $L^{2}\left(0, T ; L^{4}(\Omega)^{3}\right)$. By standard results this allows to pass to the limit in the weak formulation, showing that indeed $\mathbf{v}$ is a regular-weak solution. We skip the details. It remains to check the initial data. The weak convergence implies that for $0 \leq t \leq T$

$\|\mathbf{v}(t)\|^{2}+\alpha\|\sqrt{\ell} D \mathbf{v}(t)\|^{2}+\nu \int_{0}^{t}\|\nabla \mathbf{v}(s)\|^{2} d s \leq\|\mathbf{v}(0)\|^{2}+\alpha\|\sqrt{\ell} D \mathbf{v}(0)\|^{2}+\int_{0}^{t}\langle\mathbf{f}(s), \mathbf{v}(s)\rangle d s$.

Observe that the above inequality is obtained from (4.8), after integration in time and passing to the limit. The inequality comes from the fact that $\nabla \mathbf{v}^{n} \rightarrow \nabla \mathbf{v}$ in $L^{2}\left(0, T ; L^{2}(\Omega)^{9}\right)$, and we have to consider the inferior limit of the norm. In particular, we observe that since $\nabla \mathbf{v}^{n}(0) \rightarrow \nabla \mathbf{v}_{0}$ in $L^{2}(\Omega)$, we can suppose, up to a further sub-sequence that $\nabla \mathbf{v}^{n}(0, \mathbf{x}) \rightarrow \nabla \mathbf{v}_{0}(\mathbf{x})$ a.e. $\mathbf{x} \in \Omega$, hence using the boundedness of $\ell$ and Lebesgue dominated convergence, we have

$$
\left\|\sqrt{\ell} D \mathbf{v}^{n}(0)\right\|^{2} \rightarrow\left\|\sqrt{\ell} D \mathbf{v}_{0}\right\|^{2}
$$

showing also the correct limit at the initial time.

Step 4. Energy balance and uniqueness. We start with the energy balance (4.7). To this end one has first to justify the use of $\mathbf{v}$ as test function. From the results above, we deduce that $\mathbf{v} \otimes \mathbf{v} \in L^{\infty}\left(0, T ; L^{3}(\Omega)^{9}\right)$ which yields in particular $(\mathbf{v} \cdot \nabla) \mathbf{v} \in L^{2}\left(0, T ; V^{\prime}\right)$ and $\langle(\mathbf{v} \cdot \nabla) \mathbf{v}, \mathbf{v}\rangle=0$ according to standard results. From there, the relevant point is to check that for any $s \in[0, T]$ :

$$
\int_{0}^{s}\left(\ell D \mathbf{v}_{t}, D \mathbf{v}\right) d t=\frac{1}{2}\|\sqrt{\ell} D \mathbf{v}(s)\|^{2}-\frac{1}{2}\left\|\sqrt{\ell} D \mathbf{v}_{0}\right\|^{2},
$$


since all other terms are well-behaved due to the available regularity of v. However, $\sqrt{\ell} D \mathbf{v}, \sqrt{\ell} D \mathbf{v}_{t} \in L^{2}\left(0, T ; L^{2}(\Omega)^{9}\right)$. Therefore, by identifying $L^{2}(\Omega)^{9}$ with its dual space, we naturally have

$$
\left(\ell D \mathbf{v}_{t}, D \mathbf{v}\right)=\left\langle\sqrt{\ell} D \mathbf{v}_{t}, \sqrt{\ell} D \mathbf{v}\right\rangle=\frac{1}{2} \frac{d}{d t}\|\sqrt{\ell} D \mathbf{v}\|^{2}
$$

hence (4.15) and then (4.7) follows.

Moreover, this result allows us also to prove uniqueness of regular-weak solutions. In fact, if $\mathbf{v}_{1}$ and $\mathbf{v}_{2}$ are solutions corresponding to the same initial datum and same external force, taking the difference and testing (by the above argument this is fully justified) with $\mathbf{V}=\mathbf{v}_{1}-\mathbf{v}_{2}$ one obtains the following differential equality for the difference for any $t \in[0, T]:$

$$
\|\mathbf{V}(t)\|^{2}+\alpha\|\sqrt{\ell} D \mathbf{V}(t)\|^{2}+\nu \int_{0}^{t}\|\nabla \mathbf{V}\|^{2} d s=-\int_{0}^{t} \int_{\Omega}(\mathbf{V} \cdot \nabla) \mathbf{v}_{2} \cdot \mathbf{V} d \mathbf{x} d s .
$$

Hence, by the usual Sobolev inequalities

$$
\|\mathbf{V}(t)\|^{2}+\alpha\|\sqrt{\ell} D \mathbf{V}(t)\|^{2}+\nu \int_{0}^{t}\|\nabla \mathbf{V}\|^{2} d s \leq \frac{\nu}{2} \int_{0}^{t}\|\nabla \mathbf{V}\|^{2} d s+\frac{C}{\nu} \int_{0}^{t}\left\|\nabla \mathbf{v}_{2}\right\|^{4}\|\mathbf{V}\|^{2} d s
$$

and since $\mathbf{V}(0)=\mathbf{0}$ the Gronwall's lemma shows that $\mathbf{V} \equiv \mathbf{0}$, due to the fact that

$$
\nabla \mathbf{v}_{2} \in L^{\infty}\left(0, T ; L^{2}(\Omega)^{9}\right) \subset L^{4}\left(0, T ; L^{2}(\Omega)^{9}\right) .
$$

Remark 4.3. The pressure is not involved in Definition 4.1. However, let $\left(\mathbf{v}_{0}, \mathbf{f}\right)$ be given as in Theorem 4.1 and $\mathbf{v}$ the corresponding regular-weak solution. Then by the De Rham theorem, we easily deduce the existence of $p \in \mathcal{D}^{\prime}\left(0, T ; L^{2}(\Omega) / \mathbb{R}\right)$ such that $(\mathbf{v}, p)$ satisfies System (4.1) in the sense of the distributions. The regularity of the pressure is probably even better than that, but this point remains to be investigated.

Remark 4.4. Definition 4.1 is equivalent to the following: The field $\mathbf{v}$ is a regular-weak solution to (4.1) if:

1. $\mathbf{v} \in W^{1,2}\left(0, T ; V_{1 / 2}\right) \cap L^{\infty}(0, T ; V), \sqrt{\ell} D \mathbf{v}_{t} \in L^{2}\left(Q_{T}\right)^{9}$,

2. for all $\mathbf{w} \in L^{2}(0, T ; V), \forall s<T$ :

$$
\begin{aligned}
\int_{0}^{s}\left(\mathbf{v}_{t}, \mathbf{w}\right) d t+\alpha \int_{0}^{s}\left(\sqrt{\ell} D \mathbf{v}_{t}, \sqrt{\ell} D \mathbf{w}\right) d t & -\int_{0}^{s} \int_{\Omega} \mathbf{v} \otimes \mathbf{v}: \nabla \mathbf{w} d \mathbf{x} d t \\
& +\nu \int_{0}^{s} \int_{\Omega} \nabla \mathbf{v}: \nabla \mathbf{w} d \mathbf{x} d t=\int_{0}^{s}\langle\mathbf{f}, \mathbf{w}\rangle d t
\end{aligned}
$$

3. $\lim _{t \rightarrow 0^{+}}\left\|\mathbf{v}(t)-\mathbf{v}_{0}\right\|_{V_{1 / 2}}=0$.

Once the above results of existence and uniqueness have been proved for the generalized Navier-Stokes-Voigt equations, it is straightforward to prove the same also for the model with an additional turbulent viscosity $\nu_{\text {turb }}$ which is non-negative and bounded. We do not reproduce here the proof, but we just present the summary as follows: 
Remark 4.5. Let $\nu_{\text {turb }} \in L^{\infty}\left(\left[0, \infty[\times \Omega)\right.\right.$ such that $\nu_{\text {turb }} \geq 0$ a.e. in $[0, \infty[\times \Omega$. We consider the initial problem resulting from Section 2, with an eddy viscosity term:

$$
\left\{\begin{aligned}
\mathbf{v}_{t}-\alpha \nabla \cdot\left(\ell D \mathbf{v}_{t}\right)+(\mathbf{v} \cdot \nabla) \mathbf{v}-\nu \Delta \mathbf{v}-\nabla \cdot\left(\nu_{\text {turb }} D \mathbf{v}\right)+\nabla p=\mathbf{f} & & \text { in }(0, T) \times \Omega \\
\nabla \cdot \mathbf{v}=0 & & \text { in }(0, T) \times \Omega \\
\left.\mathbf{v}\right|_{\Gamma}=0 & & \text { on }(0, T) \times \Gamma, \\
\mathbf{v}_{t=0}=\mathbf{v}_{0} & & \text { in } \Omega .
\end{aligned}\right.
$$

We express the additional eddy viscosity term $-\nabla \cdot\left(\nu_{\text {turb }} D \mathbf{v}\right)$ by

$$
-\left\langle\nabla \cdot\left(\nu_{\text {turb }} D \mathbf{v}\right), \mathbf{w}\right\rangle=\left(\nu_{\text {turb }} D \mathbf{v}, D \mathbf{w}\right) .
$$

Regarding the conditions about $\nu_{\mathrm{turb}}$, the generalization of Theorem 4.1 to Problem (4.16) is straightforward, and $\mathbf{v}_{0} \in V$ and $\mathbf{f} \in L^{2}\left(0, T ; H^{-1 / 2}(\Omega)^{3}\right)$ being given, (4.16) has a unique regular-weak solution that satisfies the energy balance ${ }^{6}$

$$
E(t)(\alpha, \ell)+\int_{0}^{t}\left\|\left(2 \nu+\nu_{\text {turb }}\right)^{1 / 2} D \mathbf{v}(s)\right\|^{2} d s=E(0)(\alpha, \ell)+\int_{0}^{t}\langle\mathbf{f}(s), \mathbf{v}(s)\rangle d s,
$$

where again $E(t)(\alpha, \ell)=\frac{1}{2}\left(\|\mathbf{v}(t)\|^{2}+\alpha\|\sqrt{\ell} D \mathbf{v}(t)\|^{2}\right)$.

\section{Turbulent Voigt model involving the TKE}

In this section we consider the generalized Voigt model with turbulent viscosity, coupled with the equation for the turbulent kinetic energy, and in particular we prove a compactness result which allows to prove existence of weak solutions.

\subsection{A compactness Lemma}

We consider a family of models as in (4.16), associated with different realizations of the turbulent viscosity and study the behavior of the solutions, under mild conditions on the given additional viscosities.

To this end let be given $\left(\nu_{\text {turb }}^{n}\right)_{n \in \mathbb{N}}$ such that

$$
\forall n \geq 0, \quad \nu_{\text {turb }}^{n} \in L^{\infty}\left(\left[0, \infty[\times \Omega), \quad \nu_{\text {turb }}^{n} \geq 0 \text { a.e. in }[0, \infty[\times \Omega .\right.\right.
$$

Let $\mathbf{v}_{0} \in V$ and $\mathbf{f} \in L^{2}\left(0, T ; H^{-1 / 2}(\Omega)^{3}\right)$. Let $\left(\mathbf{v}^{n}, p^{n}\right)$ finally denote the distributional solution to

$$
\left\{\begin{array}{rlrl}
\mathbf{v}_{t}^{n}-\alpha \nabla \cdot\left(\ell D \mathbf{v}_{t}^{n}\right)+\left(\mathbf{v}^{n} \cdot \nabla\right) \mathbf{v}^{n}-\nu \Delta \mathbf{v}^{n}-\nabla \cdot\left(\nu_{\text {turb }}^{n} D \mathbf{v}^{n}\right)+\nabla p^{n} & =\mathbf{f} & \text { in }(0, T) \times \Omega, \\
\nabla \cdot \mathbf{v}^{n}=0 & \text { in }(0, T) \times \Omega, \\
\left.\mathbf{v}^{n}\right|_{\Gamma}=0 & \text { on }(0, T) \times \Gamma, \\
\mathbf{v}_{t=0}^{n}=\mathbf{v}_{0} & \text { in } \Omega,
\end{array}\right.
$$

and such that $\mathbf{v}^{n}$ is a regular-weak solution to (5.1).

Concerning the behavior of the solutions $\mathbf{v}^{n}$ we have the following lemma.

\footnotetext{
${ }^{6}$ Remind that since $\nabla \cdot \mathbf{v}=0$, then $\Delta \mathbf{v}=2 \nabla \cdot D \mathbf{v}$. Therefore, $\left\langle-\nu \Delta \mathbf{v}+\nabla \cdot\left(\nu_{\text {turb }} D \mathbf{v}\right), \mathbf{w}\right\rangle=((2 \nu+$ $\left.\left.\nu_{\text {turb }}\right) D \mathbf{v}, D \mathbf{w}\right)$.
} 
Lemma 5.1. Assume that the sequence $\left(\nu_{\text {turb }}^{n}\right)_{n \in \mathbb{N}}$ is uniformly bounded in $L^{\infty}([0, \infty[\times \Omega)$ and converges almost everywhere to $\nu_{\text {turb }}$ in $Q_{\infty}=[0, \infty[\times \Omega$.

Then, it follows that:

1) The sequence $\left(\mathbf{v}^{n}\right)_{n \in \mathbb{N}}$ weakly converges in $W^{1,2}\left(0, T ; V_{1 / 2}\right) \cap L^{p}(0, T ; V)$, for all $p<\infty$, to a regular-weak solution $\mathbf{v}$ of the limit problem

$$
\left\{\begin{array}{rlrl}
\mathbf{v}_{t}-\alpha \nabla \cdot\left(\ell D \mathbf{v}_{t}\right)+(\mathbf{v} \cdot \nabla) \mathbf{v}-\nu \Delta \mathbf{v}-\nabla \cdot\left(\nu_{\text {turb }} D \mathbf{v}\right)+\nabla p=\mathbf{f} & & \text { in }(0, T) \times \Omega, \\
\nabla \cdot \mathbf{v}=0 & & \text { in }(0, T) \times \Omega, \\
\left.\mathbf{v}\right|_{\Gamma}=0 & & \text { on }(0, T) \times \Gamma, \\
\mathbf{v}_{t=0} & =\mathbf{v}_{0} & & \text { in } \Omega .
\end{array}\right.
$$

2) The sequence $\left(\nu_{\text {turb }}^{n}\left|D \mathbf{v}^{n}\right|^{2}\right)_{n \in \mathbf{N}}$ converges in the sense of measures to $\nu_{\text {turb }}|D \mathbf{v}|^{2}$ in $Q_{T}$, which means that

$$
\forall \varphi \in C\left(\bar{Q}_{T}\right), \quad \int_{0}^{T} \int_{\Omega} \nu_{\text {turb }}^{n}\left|D \mathbf{v}^{n}\right|^{2} \varphi d \mathbf{x} d t \underset{n \rightarrow \infty}{\longrightarrow} \int_{0}^{T} \int_{\Omega} \nu_{\text {turb }}|D \mathbf{v}|^{2} \varphi d \mathbf{x} d t .
$$

Proof. In order to simplify the notation we extract sub-sequences, without changing the notation. However, by the uniqueness result of Theorem 4.1, we finally get convergence for the whole sequence because of the uniqueness of solutions to the limit problem.

1) As $\nu_{\text {turb }} \geq 0$ and $\nu_{\text {turb }} \in L^{\infty}$, we can repeat the proof of Theorem 4.1, which yields the existence of a unique $\mathbf{v} \in W^{1,2}\left(0, T ; V_{1 / 2}\right) \cap L^{\infty}(0, T ; V)$. Moreover, Theorem 4.1 shows also that each of the approximating problem has a unique solution $\mathbf{v}^{n}$ such that the sequence of their solutions verifies (4.14), with compactness in $L^{2}\left(0, T ; V_{3 / 4}\right)$. Passing to the limit in the equations is straightforward, except in the eddy viscosity term. To this end let be given $\mathbf{w} \in L^{2}(0, T ; V)$, we can write

$$
\left\langle-\nabla \cdot\left(\nu_{\text {turb }}^{n} D \mathbf{v}^{n}\right), \mathbf{w}\right\rangle=\int_{0}^{T} \int_{\Omega} \nu_{\text {turb }}^{n} D \mathbf{v}^{n}: D \mathbf{w} d \mathbf{x} d s=\int_{0}^{T} \int_{\Omega} D \mathbf{v}^{n}: \nu_{\text {turb }}^{n} D \mathbf{w} d \mathbf{x} d s .
$$

As $\left(\nu_{\text {turb }}^{n}\right)_{n \in \mathbb{N}}$ is bounded in $L^{\infty}\left(Q_{T}\right)$, we have on one hand the following bound

$$
\left|\nu_{\text {turb }}^{n} D \mathbf{w}\right| \leq \sup _{n \in \mathbf{N}}\left\|\nu_{\text {turb }}^{n}\right\|_{L_{t, \mathbf{x}}^{\infty}}|D \mathbf{w}| \in L^{2}\left(Q_{T}\right),
$$

and the other hand, according to the a.e convergence of $\nu_{\text {turb }}^{n}$, it follows also

$$
\nu_{\text {turb }}^{n} D \mathbf{w} \rightarrow \nu_{\text {turb }} D \mathbf{w} \quad \text { a.e in } Q_{T} .
$$

Then, by the Lebesgue dominated convergence theorem, one has that

$$
\nu_{\text {turb }}^{n} D \mathbf{w} \rightarrow \nu_{\text {turb }} D \mathbf{w} \quad \text { in } L^{2}\left(Q_{T}\right) .
$$

The convergence of the eddy viscosity term then follows from

$$
D \mathbf{v}^{n} \rightarrow D \mathbf{v} \quad \text { in } L^{2}\left(Q_{T}\right),
$$

leading to

$$
\int_{0}^{T} \int_{\Omega} D \mathbf{v}^{n}: \nu_{\text {turb }}^{n} D \mathbf{w} d \mathbf{x} d s \rightarrow \int_{0}^{T} \int_{\Omega} D \mathbf{v}: \nu_{\text {turb }} D \mathbf{w} d \mathbf{x} d s=\left\langle-\nabla \cdot\left(\nu_{\text {turb }} D \mathbf{v}\right), \mathbf{w}\right\rangle,
$$

as $n \rightarrow \infty$. As a consequence, $\mathbf{v}$ is indeed a regular-weak solution to $(5.2)$ on $[0, T]$, for all positive $T$.

2) We split the proof into three steps: 
i) Weak convergence in $L^{2}\left(Q_{T}\right)$ of the sequence $\left(\left(2 \nu+\nu_{\text {turb }}^{n}\right)^{1 / 2} D \mathbf{v}^{n}\right)_{n \in \mathrm{N}}$ to $\left(2 \nu+\nu_{\text {turb }}\right)^{1 / 2} D \mathbf{v}$;

ii) Strong convergence by the "energy method";

iii) Proof of the convergence in measures from (5.3).

i) We already proved that the sequence $\left(\left(2 \nu+\nu_{\text {turb }}^{n}\right)^{1 / 2} D \mathbf{v}^{n}\right)_{n \in \mathbb{N}}$ is bounded in $L^{2}\left(Q_{T}\right)^{9}$, uniformly in $n \in \mathbb{N}$. Moreover, we already know that $D \mathbf{v}^{n} \rightarrow D \mathbf{v}$ in $L^{2}\left(Q_{T}\right)$. Let

$$
A_{n}:=\left(2 \nu+\nu_{\text {turb }}^{n}\right)^{1 / 2} D \mathbf{v}^{n} \quad \text { and } \quad A:=\left(2 \nu+\nu_{\text {turb }}\right)^{1 / 2} D \mathbf{v}
$$

We aim to prove that $A_{n} \rightarrow A$ in $L^{2}\left(Q_{T}\right)^{9}$. To do so, let us fix $B \in L^{2}\left(Q_{T}\right)^{9}$. By the hypotheses on $\left(\nu_{\text {turb }}^{n}\right)_{n \in \mathrm{N}}$ it follows that

$$
\left(2 \nu+\nu_{\text {turb }}^{n}\right)^{1 / 2} B \rightarrow\left(2 \nu+\nu_{\text {turb }}\right)^{1 / 2} B \quad \text { a.e. in } Q_{T} .
$$

Moreover, one has also

$$
\left|\left(2 \nu+\nu_{\text {turb }}^{n}\right)^{1 / 2} B\right| \leq C\left(2 \nu+\sup _{n}\left\|\nu_{\text {turb }}^{n}\right\|_{\infty}\right)|B| \in L^{2}\left(Q_{T}\right) .
$$

Therefore, again by Lebesgue's theorem we obtain

$$
\left(2 \nu+\nu_{\text {turb }}^{n}\right)^{1 / 2} B \rightarrow\left(2 \nu+\nu_{\text {turb }}\right)^{1 / 2} B \quad \text { in } L^{2}\left(Q_{T}\right),
$$

hence

$$
\int_{0}^{T} \int_{\Omega}\left(2 \nu+\nu_{\text {turb }}^{n}\right)^{1 / 2} B: D \mathbf{v}^{n} d \mathbf{x} d t \rightarrow \int_{0}^{T} \int_{\Omega}\left(2 \nu+\nu_{\text {turb }}\right)^{1 / 2} B: D \mathbf{v} d \mathbf{x} d t
$$

yielding the desired weak convergence.

ii) Energy method. We now prove the strong $L^{2}$-convergence of the sequence $\left(A_{n}\right)_{n \in \mathbb{N}}$ to $A$. To do so, we use the energy method (see $[5,16]$ ), based on the energy (equality) balance (4.7) satisfied by both $\mathbf{v}^{n}$ and $\mathbf{v}$, with the eddy viscosity terms

$$
\iint_{Q_{t}} \nu_{\text {turb }}|D \mathbf{v}|^{2} \quad \text { and } \quad \iint_{Q_{t}} \nu_{\text {turb }}^{n}\left|D \mathbf{v}^{n}\right|^{2},
$$

in the corresponding equation. This means, to consider for all $t<T$,

$$
\left\{\begin{array}{c}
E(t)(\alpha, \ell)+\int_{0}^{t} \int_{\Omega}|A|^{2} d \mathbf{x} d s=\int_{0}^{t}\langle\mathbf{f}, \mathbf{v}\rangle d s+E(0)(\alpha, \ell), \\
E^{n}(t)(\alpha, \ell)+\int_{0}^{t} \int_{\Omega}\left|A_{n}\right|^{2} d \mathbf{x} d s=\int_{0}^{t}\left\langle\mathbf{f}, \mathbf{v}^{n}\right\rangle d s+E^{n}(0)(\alpha, \ell),
\end{array}\right.
$$

where, as usual,

$$
\begin{aligned}
E(t)(\alpha, \ell) & =\frac{1}{2}\left(\|\mathbf{v}(t)\|^{2}+\alpha\|\sqrt{\ell} D \mathbf{v}(t)\|^{2}\right), \\
E^{n}(t)(\alpha, \ell) & =\frac{1}{2}\left(\left\|\mathbf{v}^{n}(t)\right\|^{2}+\alpha\left\|\sqrt{\ell} D \mathbf{v}^{n}(t)\right\|^{2}\right) .
\end{aligned}
$$


A critical tool is that of integrating with the respect to the time variable each equation in (5.4) over $[0, T]$ and to perform then an integration by parts. This yields the following two equalities

$$
\left\{\begin{array}{c}
\int_{0}^{T} E(t)(\alpha, \ell) d t+\int_{0}^{T} \int_{\Omega}(T-t)|A|^{2} d \mathbf{x} d t=\int_{0}^{T} \int_{0}^{t}\langle\mathbf{f}, \mathbf{v}\rangle d s d t+T E(0)(\alpha, \ell) \\
\int_{0}^{T} E^{n}(t)(\alpha, \ell) d t+\int_{0}^{T} \int_{\Omega}(T-t)\left|A_{n}\right|^{2} d \mathbf{x} d t=\int_{0}^{T} \int_{0}^{t}\left\langle\mathbf{f}, \mathbf{v}^{n}\right\rangle d s d t+T E^{n}(0)(\alpha, \ell) .
\end{array}\right.
$$

Arguing with the usual compactness tools as in the proof of the previous theorems we obtain that

$$
\int_{0}^{T}\left\|\mathbf{v}^{n}(t)\right\|^{2} d t \rightarrow \int_{0}^{T}\|\mathbf{v}(t)\|^{2} d t
$$

as well as

$$
\begin{aligned}
\int_{0}^{T} \int_{0}^{t}\left\langle\mathbf{f}, \mathbf{v}^{n}\right\rangle d s d t & \rightarrow \int_{0}^{T} \int_{0}^{t}\langle\mathbf{f}, \mathbf{v}\rangle d s d t \\
T E^{n}(0)(\alpha, \ell) & \rightarrow T E(0)(\alpha, \ell),
\end{aligned}
$$

as $n \rightarrow \infty$. Therefore, by using the integrated energy equalities, we also get by comparison

$$
\int_{0}^{T} \int_{\Omega}\left[\alpha \ell\left|D \mathbf{v}^{n}\right|^{2}+(T-t)\left|A_{n}\right|^{2}\right] d \mathbf{x} d t \underset{n \rightarrow \infty}{\longrightarrow} \int_{0}^{T} \int_{\Omega}\left[\alpha \ell|D \mathbf{v}|^{2}+(T-t)|A|^{2}\right] d \mathbf{x} d t .
$$

Let now $B_{n}$ be defined as follows

$$
B_{n}:=\left(\alpha \ell+(T-t)\left(2 \nu+\nu_{\text {turb }}^{n}\right)\right)^{1 / 2} D \mathbf{v}^{n} .
$$

By the weak convergence result as in the previous steps, we immediately conclude that

$$
B_{n} \rightarrow B=\left(\alpha \ell+(T-t)\left(2 \nu+\nu_{\text {turb }}\right)\right)^{1 / 2} D \mathbf{v} \quad \text { in } L^{2}\left(Q_{T}\right)^{9},
$$

which yields the convergence of $A_{n}$ to $A$ in $L^{2}\left(Q_{T^{\prime}}\right)$ for all $T^{\prime}<T$. As $T$ can be any positive time, this concludes this step.

iii) Proof of (5.3). By the "Lebesgue inverse Theorem," since $A_{n} \rightarrow A$ in $L^{2}\left(Q_{T}\right)$, we can extract sub-sequence, still denoted by $\left(A_{n}\right)_{n \in \mathrm{N}}$, which converges to $A$ almost everywhere in $Q_{T}$, and such that there exists $G \in L^{2}\left(Q_{T}\right)$ which satisfies

$$
|A(t, \mathbf{x})| \leq G(t, \mathbf{x}) \quad \text { a.e. in } Q_{T} .
$$

Let $\varphi \in C\left(\overline{Q_{T}}\right), \varphi \geq 0$, be fixed and set

$$
B_{n}:=\sqrt{\varphi} \sqrt{\nu_{\mathrm{turb}}^{n}} D \mathbf{v}^{n} \quad \text { and } \quad B=\sqrt{\varphi} \sqrt{\nu_{\mathrm{turb}}} D \mathbf{v} .
$$

By using the definition of $A_{n}$ we get

$$
B_{n}=\sqrt{\varphi} \frac{\sqrt{\nu_{\text {turb }}^{n}}}{\left(2 \nu+\nu_{\text {turb }}^{n}\right)^{1 / 2}} A_{n} \quad \text { and } \quad B=\sqrt{\varphi} \frac{\sqrt{\nu_{\text {turb }}}}{\left(2 \nu+\nu_{\text {turb }}\right)^{1 / 2}} A .
$$

Obviously, it follows that $B_{n} \rightarrow B$ a.e. in $Q_{T}$, and by (5.5),

$$
\left|B_{n}(t, \mathbf{x})\right| \leq \frac{1}{2 \nu}\|\varphi\|_{\infty}^{1 / 2} \sup _{n}\left\|\nu_{\text {turb }}^{n}\right\|_{\infty}^{1 / 2} G(t, \mathbf{x}) \in L^{2}\left(Q_{T}\right) .
$$

Therefore, $B_{n} \rightarrow B$ in $L^{2}\left(Q_{T}\right)$, hence (5.3) follows for all non-negative $\varphi$. The proof for all $\varphi \in C\left(\overline{Q_{T}}\right)$ follows by using the splitting $\varphi=\varphi^{+}-\varphi^{-}$, where $\varphi^{+}, \varphi^{-} \geq 0$. 


\subsection{Application to the NSTKE-Voigt model}

We now apply the existence result together with the compactness lemma to study the Voigt model coupled with the equation of the turbulent kinetic energy. The NSTKE-Voigt model is in fact obtained by coupling the turbulent Navier-Stokes-Voigt equation to the equation for the TKE, following the law (2.4), which gives the following system:

$$
\begin{cases}\mathbf{v}_{t}-\alpha \nabla \cdot\left(\ell D \mathbf{v}_{t}\right)+(\mathbf{v} \cdot \nabla) \mathbf{v}-\nu \Delta \mathbf{v}-\nabla \cdot\left(\nu_{\text {turb }}(k) D \mathbf{v}\right)+\nabla p=\mathbf{f}, & (i) \\ \nabla \cdot \mathbf{v}=0, & (\text { ii }) \\ \left.\mathbf{v}\right|_{\Gamma}=0, & (\text { iii }) \\ \mathbf{v}_{t=0}=\mathbf{v}_{0}, & (i v) \\ k_{t}+\mathbf{v} \cdot \nabla k-\nabla \cdot\left(\mu_{\text {turb }}(k) \nabla k\right)=\nu_{\text {turb }}(k)|D \mathbf{v}|^{2}-(\ell+\eta)^{-1} k \sqrt{|k|}, & (v) \\ \left.k\right|_{\Gamma}=0, & (v i) \\ k_{t=0}=k_{0} . & \text { (vii) }\end{cases}
$$

This system calls for two comments:

1) According to Lemma 5.1, we know how to deal with bounded eddy viscosities and not better. This is why we cannot take the law (2.4) that we replace, as often in this class of problems, by

$$
\nu_{\text {turb }}(k)=\ell T_{N}(\sqrt{|k|}),
$$

where $T_{N}$ is the usual "truncation function" at height $N$, for a given large $N \in \mathbb{N}$, which is defined by for all $x \in \mathbb{R}$

$$
T_{N}(x):= \begin{cases}x & \text { if }|x| \leq N \\ N \frac{x}{|x|} & \text { if }|x|>N\end{cases}
$$

The eddy viscosity (5.7) has the structure of that considered in Lemma 5.1 where $\nu_{\text {turb }}(k)=\ell \rho(k)$, with $\rho(k)=T_{N}(\sqrt{|k|})$. Similarly, we assume that the viscosity coefficient for the kinetic energy satisfies

$$
\mu_{\text {turb }}(k)=C \ell T_{N^{\prime}}(\sqrt{|k|}),
$$

for some dimensionless constant $C$ and another $N^{\prime} \in \mathbb{N}$.

2) Usually, the dissipation term in the r.h.s of the equation for $k$ should be $\varepsilon:=\ell^{-1} k \sqrt{|k|}$. Unfortunately, due to the degeneration of $\ell$ at the boundary $\Gamma$, there could be further issues when passing to the limit in this term. As a precaution, we have approximated it by $\varepsilon=(\ell+\eta)^{-1} k \sqrt{|k|}$ where $\eta>0$ is a small parameter. We did not have studied yet the behavior of the solutions when $\eta \rightarrow 0$.

Theorem 5.1. Let be given $\mathbf{v}_{0} \in V$, f $\in L^{2}\left(0, T ; H^{-1 / 2}(\Omega)^{3}\right)$ and $0 \leq k_{0} \in L^{1}(\Omega)$. Assume that $\nu_{\mathrm{turb}}$ and $\mu_{\mathrm{turb}}$ are given by (5.7) and (5.8). Then there exists $(\mathbf{v}, k)$ such that:

1. The vector field $\mathbf{v}$ is a regular-weak solution to the subsystem [(5.6)-(i)-(ii)-(iii)(iv))],

2. The scalar field $k$ verifies

$$
k \in L^{\infty}\left(0, T ; L^{1}(\Omega)\right), \quad k \in \bigcap_{1<p<5 / 4} L^{p}\left(0, T ; W^{1, p}(\Omega)\right)=K_{5 / 4},
$$

and is solution of the subsystem [(5.6)-(v)-(vi)-(vii))] in the sense of the distribution in $Q_{T}$. Moreover, $k \geq 0$ a.e. in $Q_{T}$. 
Proof. System (5.6) is very close to that studied in [5, Chapter 8]. Therefore, we only indicate the changes in the proof of existence, without giving full details, which can be easily filled by the reader. The main difference is the result of the compactness Lemma 5.1, which is essential to the proof. The further (compared to the previously studied systems) regularity enforced by the generalized Voigt term is the key to prove the existence results for the full NSTKE model.

The issue is due to the quadratic source term $\nu_{\text {turb }}(k)|D \mathbf{v}|^{2}$ in the TKE equation, which is a priori in $L^{1}\left(Q_{T}\right)$ and not better. To overcome this, we truncate this term as well as the initial data at height $n \in \mathbb{N}$, leading to the following regularized system:

$$
\left\{\begin{array}{l}
\mathbf{v}_{t}-\alpha \nabla \cdot\left(\ell D \mathbf{v}_{t}\right)+(\mathbf{v} \cdot \nabla) \mathbf{v}-\nu \Delta \mathbf{v}-\nabla \cdot\left(\nu_{\text {turb }}(k) D \mathbf{v}\right)+\nabla p=\mathbf{f} \\
\nabla \cdot \mathbf{v}=0 \\
\left.\mathbf{v}\right|_{\Gamma}=0 \\
\mathbf{v}_{t=0}=\mathbf{v}_{0} \\
k_{t}+\mathbf{v} \cdot \nabla k-\nabla \cdot\left(\mu_{\mathrm{turb}}(k) \nabla k\right)=T_{n}\left(\nu_{\mathrm{turb}}(k)|D \mathbf{v}|^{2}\right)-(\ell+\eta)^{-1} k \sqrt{|k|} \\
\left.k\right|_{\Gamma}=0 \\
k_{t=0}=T_{n}\left(k_{0}\right)
\end{array}\right.
$$

For a given $\widetilde{k} \in L^{2}\left(0, T ; H_{0}^{1}(\Omega)\right) \cap L^{\infty}\left(0, T ; L^{2}(\Omega)\right)$, let $\mathbf{v}=\mathbf{v}(\widetilde{k})$ be the unique regular-weak solution to the subsystem [(5.9)-(i)-(ii)-(iii)-(iv))] with $\nu_{\text {turb }}(k)$ is replaced by $\nu_{\text {turb }}(\widetilde{k})$, so that the problem reduces to analyze the equation for $k$, considering

$$
\left\{\begin{array}{l}
k_{t}+\mathbf{v}(\widetilde{k}) \cdot \nabla k-\nabla \cdot\left(\mu_{\text {turb }}(k) \nabla k\right)=T_{n}\left(\nu_{\text {turb }}(k)|D \mathbf{v}(\widetilde{k})|^{2}\right)-(\ell+\eta)^{-1} k \sqrt{|k|} \\
\left.k\right|_{\Gamma}=0 \\
k_{t=0}=T_{n}\left(k_{0}\right)
\end{array}\right.
$$

which is a non linear parabolic equation with coefficients and a source term smooth enough which can be easily handled. The existence of a weak solution $k \in L^{2}\left(0, T ; H_{0}^{1}(\Omega)\right) \cap$ $L^{\infty}\left(0, T ; L^{2}(\Omega)\right)$ to Problem (5.10), it is easily proved and, in addition, it follows $k_{t} \in$ $L^{2}\left(0, T ; H^{-1}(\Omega)\right)$.

Finally the full system (5.9) can be solved by another application of the Leray-Schauder fixed point theorem (see [5]) and again we do not know if the resulting solution is unique. We iteratively construct the solution starting from $k^{0} \equiv 0$ and $\mathbf{v}^{0}$ the corresponding solution of the [(5.6)-(i)-(ii)-(iii)-(iv))], with $\nu_{\text {turb }}=\nu_{\text {turb }}(0)$. Then we iteratively construct the sequence of solutions along the following iterative scheme

$$
\left\{\begin{array}{l}
\mathbf{v}_{t}^{n}-\alpha \nabla \cdot\left(\ell D \mathbf{v}_{t}^{n}\right)+\left(\mathbf{v}^{n} \cdot \nabla\right) \mathbf{v}^{n}-\nu \Delta \mathbf{v}^{n}-\nabla \cdot\left(\nu_{\text {turb }}\left(k^{n}\right) D \mathbf{v}^{n}\right)+\nabla p^{n}=\mathbf{f}, \\
\nabla \cdot \mathbf{v}^{n}=0 \\
\left.\mathbf{v}^{n}\right|_{\Gamma}=0 \\
\mathbf{v}_{t=0}^{n}=\mathbf{v}_{0}, \\
k_{t}^{n}+\mathbf{v}^{n-1} \cdot \nabla k^{n}-\nabla \cdot\left(\mu_{\text {turb }}\left(k^{n}\right) \nabla k^{n}\right)=T_{n}\left(\nu_{\text {turb }}\left(k^{n}\right)\left|D \mathbf{v}^{n-1}\right|^{2}\right)-(\ell+\eta)^{-1} k^{n} \sqrt{\left|k^{n}\right|} \\
\left.k^{n}\right|_{\Gamma}=0 \\
k_{t=0}^{n}=T_{n}\left(k_{0}\right) .
\end{array}\right.
$$

We are left to pass to the limit in the above system and we know from [5, Chapter 8$]$ that, up to a sub-sequence,

$$
\left\{\begin{array}{lll}
k^{n} \rightarrow k & \text { in } \quad L^{q}\left(0, T ; W_{0}^{1, q}\right) \quad \text { for all } 1 \leq q<5 / 4, \\
k_{t}^{n} \rightarrow k_{t} & \text { in } \quad L^{q}\left(0, T ; W^{-1, q}\right) \quad \text { for all } 1 \leq q<5 / 4, \\
k^{n} \rightarrow k & \text { in } \quad L^{q}\left(Q_{T}\right) \quad \text { for all } 1 \leq q<29 / 14 \quad \text { and a.e. in } Q_{T} .
\end{array}\right.
$$


As $x \rightarrow \ell T_{N} \sqrt{|x|}$ is a continuous function over $\mathbb{R}, \nu_{\text {turb }}^{n}=\nu_{\text {turb }}\left(k^{n}\right) \rightarrow \nu_{\text {turb }}=\nu_{\text {turb }}(k)$ a.e. in $Q_{T}$, and because $\ell \in C^{1}(\bar{\Omega})$, we also have $0 \leq \nu_{\text {turb }} \leq N\|\ell\|_{\infty}$, showing that $\left(\nu_{\text {turb }}^{n}\right)_{n \in \mathbb{N}}$ verifies all the requirements of Lemma 5.1, by (5.7) and (5.12). Therefore, $\mathbf{v}^{n} \rightarrow \mathbf{v}=\mathbf{v}(k)$, the corresponding regular-weak solution to the subsystem [(5.11), (i), (ii), (iii), (iv))]. Passing to the limit in the equation for $k$ follows what is done in [5, Chapter 8], except about the quadratic source term. In this case, things are much better since, according to Lemma 5.1, $\left.T_{n}\left(\nu_{\text {turb }}\left(k^{n}\right)\left|D \mathbf{v}^{n}\right|^{2}\right) \rightarrow \nu_{\text {turb }}(k)|D \mathbf{v}|^{2}\right)$ in the sense of measures. Finally, since the presence of the truncation function obviously does not affect (5.3), this ends the proof.

\section{References}

[1] C. Amrouche, P. G. Ciarlet, L. Gratie, and S. Kesavan. On the characterizations of matrix fields as linearized strain tensor fields. J. Math. Pures Appl. (9), 86(2):116$132,2006$.

[2] L.C. Berselli and R. Lewandowski. On the Reynolds time-averaged equations and the long-time behavior of Leray-Hopf weak solutions, with applications to ensemble averages. Nonlinearity, to appear. Tech. Report on arXiv:1801.08721, 2019.

[3] L. C. Berselli, T.-Y. Kim, and L. G. Rebholz. Analysis of a reduced-order approximate deconvolution model and its interpretation as a Navier-Stokes-Voigt regularization. Discrete Contin. Dyn. Syst. Ser. B, 21(4):1027-1050, 2016.

[4] J. Boussinesq. Theorie de l'écoulement tourbillant. Mém. prés par div. savants á la Acad. Sci., 23:46-50, 1877.

[5] T. Chacón-Rebollo and R. Lewandowski. Mathematical and Numerical Foundations of Turbulence Models and Applications. Modeling and Simulation in Science, Engineering and Technology. Springer New York, 2014.

[6] P. G. Ciarlet, M. Malin, and C. Mardare. On a vector version of a fundamental lemma of J. L. Lions. Chin. Ann. Math. Ser. B, 39(1):33-46, 2018.

[7] E. Emmrich. Discrete versions of Gronwall's lemma and their application to the numerical analysis of parabolic problems. Technical report, 1999 available at

[8] P. Germain. Mécanique des milieux continus. Masson et cie, Éditeurs, Paris, 1962, 1962.

[9] V. Girault and P.-A. Raviart. Finite element methods for Navier-Stokes equations, volume 5 of Springer Series in Computational Mathematics. Springer-Verlag, Berlin, 1986. Theory and algorithms.

[10] M. E. Gurtin. An introduction to continuum mechanics, volume 158 of Mathematics in Science and Engineering. Academic Press, Inc. [Harcourt Brace Jovanovich, Publishers], New York-London, 1981.

[11] V. K. Kalantarov and E. S. Titi. Global attractors and determining modes for the 3D Navier-Stokes-Voigt equations. Chin. Ann. Math. Ser. B, 30(6):697-714, 2009.

[12] A. Larios and E. S. Titi. On the higher-order global regularity of the inviscid Voigtregularization of three-dimensional hydrodynamic models. Discrete Contin. Dyn. Syst. Ser. B, 14(2):603-627, 2010. 
[13] W. Layton and R. Lewandowski. On a well-posed turbulence model. Discrete Contin. Dyn. Syst. Ser. B, 6(1):111-128, 2006.

[14] J. Leray. Sur le mouvement d'un liquide visqueux emplissant l'espace. Acta Math., 63(1):193-248, 1934.

[15] B. Levant, F. Ramos, and E. S. Titi. On the statistical properties of the 3D incompressible Navier-Stokes-Voigt model. Commun. Math. Sci., 8(1):277-293, 2010.

[16] R. Lewandowski. The mathematical analysis of the coupling of a turbulent kinetic energy equation to the Navier-Stokes equation with an eddy viscosity. Nonlinear Anal., 28(2):393-417, 1997.

[17] R. Lewandowski. Navier-stokes equations in the whole space with an eddy viscosity. J. Math. Anal. Appl., 2019 Online first 10.1016/j.jmaa.2019.05.051

[18] R. Lewandowski, B. Pinier, E. Memin, and Chandramouli P. Testing a one-closure equation turbulence model in neutral boundary layers. Submitted. Paper avaible at hal-01875464, 2018.

[19] J.-L. Lions and E. Magenes. Non-homogeneous boundary value problems and applications. Vol. I. Springer-Verlag, New York-Heidelberg, 1972. Translated from the French by P. Kenneth, Die Grundlehren der mathematischen Wissenschaften, Band 181.

[20] J. Nečas and I. Hlaváček. Mathematical theory of elastic and elasto-plastic bodies: an introduction, volume 3 of Studies in Applied Mechanics. Elsevier Scientific Publishing Co., Amsterdam-New York, 1980.

[21] A. M. Obuhov. Turbulence in an atmosphere with inhomogeneous temperature. Akad. Nauk SSSR. Trudy Inst. Teoret. Geofiz., 1:95-115, 1946.

[22] A. P. Oskolkov. The uniqueness and solvability in the large of boundary value problems for the equations of motion of aqueous solutions of polymers. Zap. Naučn. Sem. Leningrad. Otdel. Mat. Inst. Steklov. (LOMI), 38:98-136, 1973. Boundary value problems of mathematical physics and related questions in the theory of functions, 7 .

[23] A. P. Oskolkov. On the theory of Voight fluids. Zap. Nauchn. Sem. Leningrad. Otdel. Mat. Inst. Steklov. (LOMI), 96:233-236, 310, 1980. Boundary value problems of mathematical physics and related questions in the theory of functions, 12.

[24] L. Prandtl. Prandtl-Essentials of fluid mechanics, volume 158 of Applied Mathematical Sciences. Springer, New York, third edition, 2010. Translated from the 12th German edition by Katherine Asfaw and edited by Herbert Oertel, With contributions by P. Erhard, D. Etling, U. Müller, U. Riedel, K. R. Sreenivasan and J. Warnatz.

[25] F. Ramos and E. S. Titi. Invariant measures for the 3D Navier-Stokes-Voigt equations and their Navier-Stokes limit. Discrete Contin. Dyn. Syst., 28(1):375-403, 2010.

[26] Y. Rong, W.J. Layton, and H. Zhao. Extension of a simplified Baldwin-Lomax model to nonequilibrium turbulence: Model, analysis and algorithms. Numer. Methods Partial Differential Equations, 52019.

[27] R. Temam. Navier-Stokes equations. AMS Chelsea Publishing, Providence, RI, 2001. Theory and numerical analysis, Reprint of the 1984 edition. 
[28] E. R. Van Driest. On turbulent flow near a wall. J. Aeronaut. Sci., 23(11):1007-1011, 1956.

[29] W. Walter. Ordinary differential equations, volume 182 of Graduate Texts in Mathematics. Springer-Verlag, New York, 1998. Translated from the sixth German (1996) edition by Russell Thompson, Readings in Mathematics. 